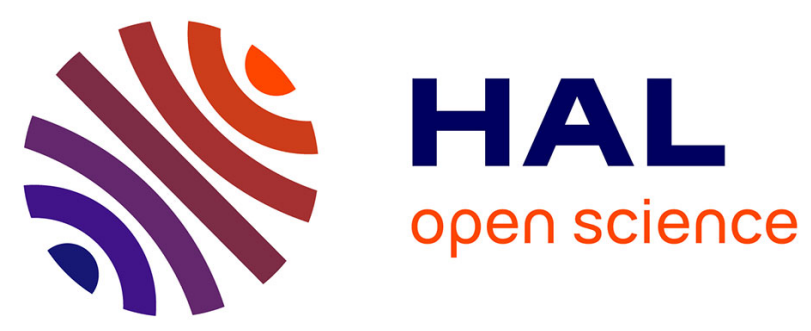

\title{
LEVEL SET-BASED SHAPE OPTIMIZATION APPROACH FOR SHARP-INTERFACE RECONSTRUCTIONS IN TIME-DOMAIN FULL WAVEFORM INVERSION
}

Yuri Flores Albuquerque, Antoine Laurain, Irwin Yousept

\section{To cite this version:}

Yuri Flores Albuquerque, Antoine Laurain, Irwin Yousept. LEVEL SET-BASED SHAPE OPTIMIZATION APPROACH FOR SHARP-INTERFACE RECONSTRUCTIONS IN TIME-DOMAIN FULL WAVEFORM INVERSION. 2020. hal-03049006

\section{HAL Id: hal-03049006 https://hal.science/hal-03049006}

Preprint submitted on 9 Dec 2020

HAL is a multi-disciplinary open access archive for the deposit and dissemination of scientific research documents, whether they are published or not. The documents may come from teaching and research institutions in France or abroad, or from public or private research centers.
L'archive ouverte pluridisciplinaire HAL, est destinée au dépôt et à la diffusion de documents scientifiques de niveau recherche, publiés ou non, émanant des établissements d'enseignement et de recherche français ou étrangers, des laboratoires publics ou privés. 


\title{
LEVEL SET-BASED SHAPE OPTIMIZATION APPROACH FOR SHARP-INTERFACE RECONSTRUCTIONS IN TIME-DOMAIN FULL WAVEFORM INVERSION
}

\author{
YURI FLORES ALBUQUERQUE, ANTOINE LAURAIN, AND IRWIN YOUSEPT
}

\begin{abstract}
Velocity models presenting sharp interfaces are highly relevant in seismic imaging, for instance for imaging the subsurface of the Earth in the presence of salt bodies. In order to mitigate the oversmoothing of classical regularization strategies such as the Tikhonov regularization, we propose a shape optimization approach for the sharp-interface reconstruction in time-domain acoustic full waveform inversion. Our main result includes the shape differentiability of the cost functional measuring the misfit between observed and predicted data. In particular, it reveals the expression of the distributed shape derivative in tensor form, built on a Lagrangian-type approach and regularity results for the wave equation with discontinuous coefficients. Based on the achieved distributed shape derivative and the level set method, we propose a numerical approach and present several numerical tests supporting our approach.
\end{abstract}

\section{INTRODUCTION}

Seismic imaging is a set of techniques to produce images of the subsurface of the Earth. Seismic waves generated by controlled sources at the surface propagate into the medium and reflections occur at the transitions between different materials; the reflection waves are then recorded at a set of point receivers, either located at the surface or in depth. Important applications of seismic imaging include hydrocarbon exploration and imaging of the lithosphere, glaciers, and subsurface structures in volcanic areas. Full waveform inversion (FWI) is a recent variant of seismic tomography that uses the full wavefield information for the inversion, instead of simpler informations such as travel times and phase velocities, by iteratively minimizing the difference between synthetic and observed data; see [16. 45 for an overview. FWI relies on the numerical solution of the acoustic or elastic wave equations to obtain a realistic modeling of seismic wave propagation through heterogeneous media; thus FWI can be formulated as an optimization problem with PDE constraints.

The ill-posed nature of seismic inversion requires the use of a regularization. Due to its smoothness and ease of use, the Tikhonov regularization is probably the most frequently used regularization scheme for inverse problems and particularly for FWI. Its main drawback is that it tends to produce smooth velocity models, which precludes the reconstruction of singular features such as sharp interfaces, discontinuities, and high contrasts in the model. Such sharp features are nonetheless crucial for certain applications. For instance in hydrocarbon exploration, sound waves travel with greater velocity inside salt bodies compared to the neighboring sediments. Salt bodies also present a sharp velocity contrast to the sediment velocities at their boundaries and irregular geometries so that an accurate representation of the interface may considerably improve the quality of the images. Thus, an alternative regularization preserving sharp features and discontinuities is beneficial for the inversion. For this purpose the total variation regularization is widely used in image processing and has been applied to seismic imaging; see 1. 15] and the references therein. Another possible approach is to incorporate prior information about sharp interfaces and high contrast explicitly in the modeling of the problem; this has a regularization effect which alleviates the ill-posedness of the problem without oversmoothing the solution. Another possible advantage of the sharp-interface assumption is the sparse representation, which is useful in the context of large-scale applications.

In this work, we propose the use of geometric optimization techniques (cf. [42]) for the reconstruction of sharp interfaces in the coefficients (wave speed and mass density) of the acoustic wave equations. We consider the reconstruction of salt bodies and assume that the velocity models are piecewise constant for simplicity, with known constant but distinct values in the salt body and in the sediment region. In this

1991 Mathematics Subject Classification. 35Q93, 35R30, 35R05.

Key words and phrases. Full waveform inversion, acoustic wave equation, sharp interfaces, shape optimization, distributed shape derivative, level set method. 
way, the optimization problem is recast as a shape optimization problem where the interface of the salt region becomes the unknown. The problem is formulated as the minimization with respect to the interface geometry of a cost functional measuring the misfit between the velocity model and the observed data, and the evolution of the interface is performed using a level set method (see [30,38]).

FWI can be considered either in the frequency domain or in the time domain. Shape optimization (design problems) and level set-based approaches have been developed recently for FWI in the frequency domain, see $13,17,18,20,22,23,26,32,33,41,46$, but are lacking in the time domain. Our work (see also [2]) seems to be the first to develop and perform a mathematical analysis of a level set-based shape optimization approach for time-domain FWI in the acoustic case; see also 27. for a recent contribution using a level-set approach based on reaction-diffusion equation.

One of the main challenges arising in the shape optimization framework is the low regularity of the solution to the wave equations caused by the discontinuity of the wave speed and the mass density which affects both the state and adjoint state. The existing literature on shape optimization problems for the wave equation often assumes smooth coefficients; see [9, 10, 37, and [34 for an optimal design problem in the context of exact controllability for the $2 \mathrm{D}$ wave equation with an internal control. Optimal design problems have also been considered for stabilization of the wave equation involving a discontinuous damping potential in [19, 35, 36, and for exact controllability of the wave equation in [40. The uniqueness and stability of the inverse problem for time-harmonic elastic waves with piecewise constant Lamé parameters and density has been studied in [6], and the reconstruction of small conductivity inhomogeneities for the scalar wave equation has been investigated in 3]. In PDE-constrained optimization, there are a few contributions on inverse problems governed by the acoustic wave equation with discontinuous coefficients, and on the Fréchet differentiability of the parameter-to-solution map, see $[5,24,43]$ and $[25,31$ in the elastic case. In [7, 8, a semismooth Newton-CG method for constrained parameter identification is studied. We also refer to [12] for a recent study of the multi-bang control approach for the reconstruction of the mass density in the scalar wave equations.

To show the global well-posedness and the regularity properties of the corresponding state and adjoint state, we make use of the semigroup theory in combination with elliptic regularity results and techniques by Ball [4]. The semigroup theory is particularly suitable to deal with non-smooth coefficients and the hyperbolic first-order structure of the PDE-model (see, e.g., [11, 25, 47, 48]). Besides the discontinuity of the coefficients, the possibly low regularity of the interfaces is also challenging for the shape optimization approach. Indeed, the boundary expression of the shape derivative, also known as Hadamard formula, which is commonly used in the shape optimization approach, see for instance 17, 18, 41, usually requires a certain regularity of the boundary. Here, we propose a method based on a weak form of the shape derivative also called distributed shape derivative, which allows working with nonsmooth domains and functions with low regularity and often offers better accuracy than the boundary expression for numerical approximation; see $14,21,29,30$ and the references therein. The proof of the shape differentiability and the calculation of the distributed shape derivative are achieved using the averaged adjoint method introduced in [44, which is a Lagrangian-type approach for shape optimization problems; see also 30. The shape derivative depends on the time and space derivatives of the state and the adjoint state where the adjoint is the solution of a backwards wave equation with terminal conditions. Based on the distributed shape derivative and on the level set method, we eventually propose a numerical algorithm for the minimization of the cost functional. The efficiency of the algorithm is demonstrated through several examples of reconstruction with synthetic data.

The remainder of this paper is organized as follows. In Section 2, we give regularity results for the wave equation with discontinuous coefficients, which is a key ingredient for proving the shape differentiability. In Section 3 the shape optimization framework is recalled. In Section 4, we recall the averaged adjoint method (AAM) of 44 and compute the adjoint. In Section 5, we apply the AAM to compute the distributed shape derivative of the cost function. Thereafter, in Section 6, the application of our theoretical results to FWI is presented. Finally, in Section 7 we show the numerical algorithm and present several numerical results supporting our approach. 


\section{Regularity Results FOR The WAVE EQUATION WITH DisCONTINUOUS COEFFiCIENTS}

In this section we give several well-posedness and regularity results for the solution of the acoustic wave equations with damping and discontinuous coefficients. These results are essential for the study of the shape differentiability in the subsequent sections. Let $\mathcal{D} \subset \mathbb{R}^{n}, n \geq 2$, be a bounded Lipschitz domain. Let $\Gamma \subset \partial \mathcal{D}$ be a connected subset of $\partial \mathcal{D}$ where the homogeneous Dirichlet condition is imposed. On $\Gamma_{n}:=\partial \mathcal{D} \backslash \Gamma$, we impose the homogeneous Neumann condition. Let $H_{\Gamma}^{1}(\mathcal{D})$ be the closed subspace of $H^{1}(\mathcal{D})$ of functions with vanishing trace on $\Gamma$. Furthermore, we introduce the following Hilbert spaces:

$X:=L^{2}\left((0, T), H_{\Gamma}^{1}(\mathcal{D})\right) \cap H^{1}\left((0, T), L^{2}(\mathcal{D})\right), \quad X_{0}:=\{\psi \in X \mid \psi(0)=0\}, \quad X_{T}:=\{\psi \in X \mid \psi(T)=0\}$, endowed with the scalar product

$$
(u, v)_{X}:=\int_{0}^{T} u v+u_{t} v_{t}+\nabla u \cdot \nabla v
$$

and the associated norm $\|\cdot\|_{X}:=\sqrt{(\cdot, \cdot)_{X}}$.

Let us consider the following second-order wave equations:

$$
\begin{aligned}
\kappa u_{t t}-\operatorname{div}(R \nabla u)+\eta u_{t} & =f \text { in } \mathcal{D} \times(0, T), \\
u(0) & =0 \text { in } \mathcal{D}, \\
u_{t}(0) & =0 \text { in } \mathcal{D}, \\
u & =0 \text { on } \Gamma \times(0, T), \\
R \nabla u \cdot n & =0 \text { on } \Gamma_{n} \times(0, T),
\end{aligned}
$$

where the material parameters $\kappa: \mathcal{D} \rightarrow \mathbb{R}, R: \mathcal{D} \rightarrow \mathbb{R}^{n \times n}$ and $\eta: \mathcal{D} \rightarrow \mathbb{R}$ satisfy the following assumptions:

Assumption 1. Let $\kappa \in L^{\infty}(\mathcal{D}), \eta \in L^{\infty}(\mathcal{D})$ and $R \in L^{\infty}\left(\mathcal{D}, \mathbb{R}^{n \times n}\right)$. There exist positive constants $0<\underline{\kappa}<\bar{\kappa}$ such that $\underline{\kappa} \leq \kappa(x) \leq \bar{\kappa}$ hold true for a.e. $x \in \Omega$. Moreover, $\eta$ is nonnegative, and $R$ is symmetric and uniformly positive definite, i.e., there exists a positive constant $\underline{R}>0$ such that

$$
\xi^{T} R(x) \xi \geq \underline{R}|\xi|^{2} \quad \text { for all } \xi \in \mathbb{R}^{n} \text { and a.e. } x \in \mathbb{R} \text {. }
$$

A function $u \in \mathcal{C}\left([0, T], H_{\Gamma}^{1}(\mathcal{D})\right) \cap \mathcal{C}^{1}\left([0, T], L^{2}(\mathcal{D})\right)$ is called a mild solution to the hyperbolic forward problem (1)-(5) if and only if

$$
\left\{\begin{array}{r}
\frac{d}{d t} \int_{\mathcal{D}} \kappa u_{t}(t) \psi d x+\int_{\mathcal{D}} R \nabla u(t) \cdot \nabla \psi+\eta u_{t}(t) \psi d x=\int_{\mathcal{D}} f(t) \psi d x \\
\text { for all } \psi \in H_{\Gamma}^{1}(\mathcal{D}) \text { and a.e. } t \in(0, T), \\
u(0)=u_{t}(0)=0,
\end{array}\right.
$$

and for every $\psi \in H_{\Gamma}^{1}(\mathcal{D})$ the mapping $t \mapsto\left(\kappa u_{t}(t), \psi\right)_{L^{2}(\mathcal{D})}$ is absolutely continuous.

Theorem 1. If Assumption 1 is satisfied, then for every $f \in L^{1}\left((0, T), L^{2}(\mathcal{D})\right)$, the hyperbolic forward problem (1)-(5) admits a unique mild solution $u \in \mathcal{C}\left([0, T], H_{\Gamma}^{1}(\mathcal{D})\right) \cap \mathcal{C}^{1}\left([0, T], L^{2}(\mathcal{D})\right)$ satisfying

$$
\begin{aligned}
\int_{\mathcal{D}} \kappa u^{2}(t) d x & \leq t \int_{0}^{t}\left\|\kappa^{\frac{1}{2}} f(\sigma)\right\|_{L^{2}(\mathcal{D})} d \sigma \quad \forall t \in[0, T], \\
\left(\int_{\mathcal{D}} \kappa u_{t}^{2}(t) d x+\int_{\mathcal{D}} R \nabla u(t) \cdot \nabla u(t) d x\right)^{\frac{1}{2}} & \leq \int_{0}^{t}\left\|\kappa^{\frac{1}{2}} f(\sigma)\right\|_{L^{2}(\mathcal{D})} d \sigma \quad \forall t \in[0, T] .
\end{aligned}
$$

Proof. Uniqueness: Suppose that $u^{(1)}, u^{(2)} \in \mathcal{C}\left([0, T], H_{\Gamma}^{1}(\mathcal{D})\right) \cap \mathcal{C}^{1}\left([0, T], L^{2}(\mathcal{D})\right)$ satisfy (7). Then, the difference $d:=u^{(1)}-u^{(2)}$ satisfies

$$
\frac{d}{d t} \int_{\mathcal{D}} \kappa d_{t}(t) d(t) d x+\int_{\mathcal{D}} R \nabla d(t) \cdot \nabla d(t)+\eta d_{t}(t) d(t) d x=0 \quad \text { for a.e. } t \in(0, T) .
$$

Using the fact that $\int_{\mathcal{D}} \eta d_{t}(t) d(t) d x=\frac{1}{2} \frac{d}{d t}\left\|\eta^{\frac{1}{2}} d(t)\right\|_{L^{2}(\mathcal{D})}^{2}$ and $d(0)=0$, we obtain by integrating the above equality over the time interval $[0, \tau]$ for any $0 \leq \tau \leq T$ that

$$
\int_{\mathcal{D}} \kappa d_{t}(\tau) d(\tau) d x+\int_{0}^{\tau} \int_{\mathcal{D}} R \nabla d(t) \cdot \nabla d(t) d x d t+\frac{1}{2}\left\|\eta^{\frac{1}{2}} d(\tau)\right\|_{L^{2}(\mathcal{D})}^{2}=0 .
$$


Thus, in view of (6), it follows that

$$
\frac{d}{d t}\left\|\kappa^{\frac{1}{2}} d(t)\right\|_{L^{2}(\mathcal{D})}^{2}=\int_{\mathcal{D}} \kappa d_{t}(t) d(t) d x \leq 0 \quad \forall t \in[0, T] \underbrace{\Longrightarrow}_{d(0)=0} d \equiv 0 .
$$

Existence: We introduce the Hilbert spaces

$$
\begin{aligned}
H(\operatorname{div}) & :=\left\{v \in L^{2}(\mathcal{D})^{n} \mid \operatorname{div} v \in L^{2}(\mathcal{D})\right\}, \\
H_{\Gamma}(\operatorname{div}) & :=\left\{v \in H(\operatorname{div}) \mid(\operatorname{div} v, \varphi)_{L^{2}(\mathcal{D})}=-(v, \nabla \varphi)_{L^{2}(\mathcal{D})^{n}} \forall \varphi \in H_{\Gamma}^{1}(\mathcal{D})\right\},
\end{aligned}
$$

where the divergence is understood in the distributional sense. Furthermore, let $\mathcal{H}:=L^{2}(\mathcal{D}) \times L^{2}(\mathcal{D})^{n}$ equipped with the scalar product

$$
\left(\left(u_{1}, v_{1}\right),\left(u_{2}, v_{2}\right)\right)_{\mathcal{H}}:=\left(\kappa u_{1}, u_{2}\right)_{L^{2}(\mathcal{D})}+\left(R^{-1} v_{1}, v_{2}\right)_{L^{2}(\mathcal{D})^{n}} .
$$

Using these Hilbert spaces, we introduce the densely defined (unbounded) operator:

$$
\mathcal{A}: D(\mathcal{A}) \subset \mathcal{H} \rightarrow \mathcal{H}, \mathcal{A}(u, v):=-\left(\kappa^{-1}(\operatorname{div} v+\eta u), R \nabla u\right)
$$

with the effective domain $D(\mathcal{A}):=H_{\Gamma}^{1}(\mathcal{D}) \times H_{\Gamma}($ div $)$. By definition, it holds for all $(u, v) \in D(\mathcal{A})$ that

$$
(\mathcal{A}(u, v),(u, v))_{\mathcal{H}} \underbrace{=}_{[11) \&[12}(-\operatorname{div} v-\eta u, u)_{L^{2}(\mathcal{D})}-(v, \nabla u)_{L^{2}(\mathcal{D})^{n} \underbrace{=}_{110}}^{=}-(\eta u, u)_{L^{2}(\mathcal{D})} \leq 0
$$

since $\eta$ is nonnegative. In other words, $\mathcal{A}: D(\mathcal{A}) \subset \mathcal{H} \rightarrow \mathcal{H}$ is dissipative. Let us now show that the operator $\mathcal{A}-I: D(\mathcal{A}) \rightarrow \mathcal{H}$ is surjective. To this aim, let $(g, q) \in \mathcal{H}$. By the Lax-Milgram lemma, there exists a unique $u \in H_{\Gamma}^{1}(\mathcal{D})$ such that

$$
(R \nabla u, \nabla \varphi)_{L^{2}(\mathcal{D})^{n}}+((\eta+\kappa) u, \varphi)_{L^{2}(\mathcal{D})}=-(\kappa g, \varphi)_{L^{2}(\mathcal{D})}-(q, \nabla \varphi)_{L^{2}(\mathcal{D})^{n}} \quad \forall \varphi \in H_{\Gamma}^{1}(\mathcal{D}) .
$$

Making use of the solution to [14], we set

$$
v:=-(q+R \nabla u) \in L^{2}(\mathcal{D})^{n} .
$$

In view of (14), the vector field $v$ satisfies

$$
(v, \nabla \varphi)_{L^{2}(\mathcal{D})^{n}}=((\eta+\kappa) u+\kappa g, \varphi) \quad \forall \varphi \in \mathcal{C}_{0}^{\infty}(\mathcal{D}),
$$

and so the distributional definition of the divergence yields that $v \in H(\operatorname{div})$ and

$$
\operatorname{div} v=-(\eta+\kappa) u-\kappa g \text {. }
$$

Furthermore, from (14)- 16 , we also have that

$$
(\operatorname{div} v, \varphi)_{L^{2}(\mathcal{D})}=-(v, \nabla \varphi)_{L^{2}(\mathcal{D})^{n}} \quad \forall \varphi \in H_{\Gamma}^{1}(\mathcal{D}) \quad \Rightarrow \quad v \in H_{\Gamma}(\operatorname{div}) .
$$

Altogether, 12, (15) and 16) lead to the desired surjectivity result:

$$
\forall(g, q) \in \mathcal{H}, \exists(u, v) \in D(\mathcal{A}):(\mathcal{A}-I)(u, v)=(g, q) .
$$

Thanks to (13) and (17), the Lumer-Phillips theorem [39, Theorem 4.3] implies that the operator $\mathcal{A}$ : $D(\mathcal{A}) \subset \mathcal{H} \rightarrow \mathcal{H}$ generates a contraction semigroup $\left\{\mathbb{T}_{t}\right\}_{t \geq 0}$. Making use of this semigroup, we introduce

$$
(u, v)(t):=\int_{0}^{t} \mathbb{T}_{t-\sigma}(F(\sigma), 0) d \sigma \quad \forall t \in[0, T], \quad F(\sigma):=\int_{0}^{\sigma} f(\xi) d \xi \quad \forall \sigma \in[0, T] .
$$

Since $(F, 0) \in W^{1,1}((0, T), \mathcal{H})$, classical arguments yield that $(u, v) \in \mathcal{C}([0, T], D(\mathcal{A})) \cap \mathcal{C}^{1}([0, T], \mathcal{H})$ is the unique solution to

$$
\left\{\begin{aligned}
\left(\frac{d}{d t}-\mathcal{A}\right)(u, v)(t) & =(F(t), 0) \quad \forall t \in[0, T] \quad \underbrace{\Longrightarrow}_{\mid 12} v_{t}+R \nabla u=0, \\
(u, v)(0) & =(0,0),
\end{aligned}\right.
$$

and

$$
\left(u_{t}, v_{t}\right)(t)=\int_{0}^{t} \mathbb{T}_{t-\sigma}(f(\sigma), 0) d \sigma \quad \forall t \in[0, T]
$$


where we have used the fact that $F^{\prime}=f$ and $F(0)=0$ to obtain the above variation of constants formula. Then, applying the classical result by Ball 4 to 20 , it follows that $\left(u_{t}, v_{t}\right)$ satisfies

$$
\left\{\begin{array}{l}
\frac{d}{d t}\left(\left(u_{t}, v_{t}\right)(t),(\varphi, z)\right)_{\mathcal{H}}-\left(\left(u_{t}, v_{t}\right)(t), \mathcal{A}^{*}(\varphi, z)\right)_{\mathcal{H}} \\
=\left(\left(\kappa^{-1} f(t), 0\right),(\varphi, z)\right)_{\mathcal{H}} \quad \text { for all }(\varphi, z) \in D\left(\mathcal{A}^{*}\right) \text { and a.e. } t \in(0, T), \\
\left(u_{t}, v_{t}\right)(0)=(0,0),
\end{array}\right.
$$

and the mapping $t \mapsto\left(\left(u_{t}, v_{t}\right)(t),(\varphi, z)\right)_{\mathcal{H}}$ is absolutely continuous. On the other hand, in view of 12 and [10, we have that $H_{\Gamma}^{1}(\mathcal{D}) \times\{0\} \subset D(\mathcal{A}) \subset D\left(\mathcal{A}^{*}\right)$ and

$$
\begin{array}{r}
-\left(\left(u_{t}, v_{t}\right)(t), \mathcal{A}^{*}(\varphi, 0)\right)_{\mathcal{H}}=\left(u_{t}(t), \eta \varphi\right)_{L^{2}(\mathcal{D})}-\left(v_{t}(t), \nabla \varphi\right)_{L^{2}(\mathcal{D})^{n}} \\
\underbrace{=}_{[19} \int_{\mathcal{D}} R \nabla u(t) \cdot \nabla \varphi+\eta u_{t}(t) \varphi d x \quad \forall \varphi \in H_{\Gamma}^{1}(\mathcal{D}) .
\end{array}
$$

Therefore, considering $z=0$ and $\varphi \in H_{\Gamma}^{1}(\mathcal{D})$ in (21), we conclude from (22) that $u$ satisfies (7). It remains to prove that $u$ satisfies the stability estimates $(8)-(9)$. Since $\left\{\mathbb{T}_{t}\right\}_{t \geq 0}$ is a contraction semigroup, the variation of constants formula 18 implies

$$
\int_{\mathcal{D}} \kappa u^{2}(t) d x \underbrace{\leq}_{[11]}\|(u, v)(t)\|_{\mathcal{H}} \underbrace{\leq}_{[18} \int_{0}^{t} \int_{0}^{\sigma}\left\|\kappa^{\frac{1}{2}} f(\xi)\right\|_{L^{2}(\mathcal{D})} d \xi d \sigma \quad \forall t \in[0, T],
$$

which immediately yields the desired estimate (8). Similarly, the second estimate (9) is obtained as follows:

$$
\begin{aligned}
\left(\int_{\mathcal{D}} \kappa u_{t}^{2}(t) d x+\int_{\mathcal{D}} R \nabla u(t) \cdot \nabla u(t) d x\right)^{\frac{1}{2}} \underbrace{=}_{\sqrt[190]{1}}\left(\int_{\mathcal{D}} \kappa u_{t}^{2}(t) d x+\int_{\mathcal{D}} R^{-1} v_{t}(t) \cdot v_{t}(t) d x\right)^{\frac{1}{2}} \\
\underbrace{=}_{\underbrace{=}_{110}}\left\|\left(u_{t}, v_{t}\right)(t)\right\|_{\mathcal{H}} \underbrace{\leq}_{[20} \int_{0}^{t}\left\|\kappa^{\frac{1}{2}} f(\sigma)\right\|_{L^{2}(\mathcal{D})} d \sigma \quad \forall t \in[0, T] .
\end{aligned}
$$

This completes the proof.

Corollary 1. Let Assumption 1 be satisfied and $f \in W^{1,1}\left((0, T), L^{2}(\mathcal{D})\right)$. Then, the unique mild solution $u \in \mathcal{C}\left([0, T], H_{\Gamma}^{1}(\mathcal{D})\right) \cap \mathcal{C}^{1}\left([0, T], L^{2}(\mathcal{D})\right)$ of the forward hyperbolic problem (1)-(5) satisfies the higher regularity property $u \in \mathcal{C}^{2}\left([0, T], L^{2}(\mathcal{D})\right)$ and

$$
\kappa u_{t t}(t)-\operatorname{div}(R \nabla u(t))+\eta u_{t}(t)=f(t) \quad \forall t \in[0, T],
$$

i.e., it is the strong solution to (1) - (5).

Proof. Again by classical arguments, since $f \in W^{1,1}\left((0, T), L^{2}(\mathcal{D})\right)$, the variation of constants formula $(20)$ implies that $u_{t} \in \mathcal{C}^{1}\left([0, T], L^{2}(\mathcal{D})\right)$. Applying this higher regularity property to the variational equality (7) yields that

$$
\int_{\mathcal{D}} R \nabla u(t) \cdot \nabla \varphi d x=\int_{\mathcal{D}}\left(f(t)-\eta u_{t}(t)-\kappa u_{t t}(t)\right) \varphi d x \quad \forall \varphi \in H_{\Gamma}^{1}(\mathcal{D}) \quad \forall t \in[0, T] .
$$

Thus, as $\mathcal{C}_{0}^{\infty}(\mathcal{D}) \subset H_{\Gamma}^{1}(\mathcal{D})$, the distributional definition of the divergence implies that

$$
R \nabla u(t) \in H(\text { div }) \quad \text { and } \quad \operatorname{div}(R \nabla u(t))=-f(t)+\eta u_{t}(t)+\kappa u_{t t}(t) \quad \forall t \in[0, T] .
$$

In conclusion, $u$ is the strong solution to [1)-(5).

Assumption 2. Suppose that there exists an open set $\mathcal{O} \subset \mathcal{D}$ such that

$$
R(x)=r(x) I_{n} \quad \forall x \in \mathcal{O}
$$

holds for the identity matrix $I_{n} \in \mathbb{R}^{n \times n}$ and a Lipschitz-continuous function $r \in \mathcal{C}^{0,1}(\overline{\mathcal{O}})$. Moreover, there exists a positive constant $\underline{r}>0$ such that $r(x) \geq \underline{r}$ holds for all $x \in \overline{\mathcal{O}}$. 
Corollary 2. Let Assumptions 1 and 2 hold. Then, for every $f \in W^{1,1}\left((0, T), L^{2}(\mathcal{D})\right)$, the unique mild solution $u$ of the forward hyperbolic problem (1)-(5) satisfies

$$
u \in \mathcal{C}\left([0, T], H^{2}(\omega)\right)
$$

for every open set $\omega \subset \mathcal{O} \subset \mathcal{D}$ satisfying $\bar{\omega} \subset \mathcal{O}$.

Proof. Let $\omega \subset \mathcal{O}$ be an open set satisfying $\bar{\omega} \subset \mathcal{O}$. The classical interior elliptic regularity result implies that for any $y \in H^{1}(\mathcal{O})$ and $z \in L^{2}(\mathcal{O})$ satisfying

$$
-\Delta y=z \quad \text { in } \mathcal{O} \text { (in the weak sense) }
$$

it holds that $y \in H^{2}(\omega)$. Furthermore, there exists a constant $C$, depending only on $\omega$ and $\mathcal{O}$, such that

$$
\|y\|_{H^{2}(\omega)} \leq C\left(\|z\|_{L^{2}(\mathcal{O})}+\|y\|_{L^{2}(\mathcal{O})}\right) .
$$

Now, according to Corollary 1. the unique mild solution $u \in \mathcal{C}\left([0, T], H_{\Gamma}^{1}(\mathcal{D})\right) \cap \mathcal{C}^{2}\left([0, T], L^{2}(\mathcal{D})\right)$ and $f \in$ $W^{1,1}\left((0, T), L^{2}(\mathcal{D})\right) \hookrightarrow \mathcal{C}\left([0, T], L^{2}(\mathcal{D})\right)$ satisfy for every fixed $t \in[0, T]$ the elliptic problem

$$
-\operatorname{div}(R \nabla u(t))=f(t)-\eta u_{t}(t)-\kappa u_{t t}(t) \text { in } \mathcal{D},
$$

and so by Assumption 2 it follows that

$$
-\Delta u(t)=r^{-1}\left(\nabla r \cdot \nabla u(t)+f(t)-\eta u_{t}(t)-\kappa u_{t t}(t)\right) \text { in } \mathcal{O} \quad \forall t \in[0, T] .
$$

As the right hand side of $(27)$ belongs to $L^{2}(\mathcal{O})$, it follows that $u(t) \in H^{2}(\omega)$ for all $t \in[0, T]$. To prove the uniform regularity in $\mathcal{C}\left([0, T], H^{2}(\omega)\right)$, let $t, \tau \in[0, T]$ be arbitrarily fixed. By the superposition principle, (27) yields that

$$
-\Delta(u(t)-u(\tau))=r^{-1}\left(\nabla r \cdot \nabla(u(t)-u(\tau))+f(t)-f(\tau)-\eta\left(u_{t}(t)-u_{t}(\tau)\right)-\kappa\left(u_{t t}(t)-u_{t t}(\tau)\right)\right) \text { in } \mathcal{O} .
$$

Consequently, the a priori estimate 26 implies that

$$
\begin{aligned}
\|u(t)-u(\tau)\|_{H^{2}(\omega)} \leq C\left(\| r^{-1}(\nabla r \cdot \nabla(u(t)-u(\tau))\right. & +f(t)-f(\tau)-\eta\left(u_{t}(t)-u_{t}(\tau)\right) \\
& \left.\left.-\kappa\left(u_{t t}(t)-u_{t t}(\tau)\right)\right)\left\|_{L^{2}(\mathcal{O})}+\right\| u(t)-u(\tau) \|_{L^{2}(\mathcal{O})}\right) .
\end{aligned}
$$

Finally, applying the regularity $u \in \mathcal{C}\left([0, T], H_{\Gamma}^{1}(\mathcal{D})\right) \cap \mathcal{C}^{2}\left([0, T], L^{2}(\mathcal{D})\right)$ and $f \in W^{1,1}\left((0, T), L^{2}(\mathcal{D})\right) \hookrightarrow$ $\mathcal{C}\left([0, T], L^{2}(\mathcal{D})\right)$ to $[28$, we deduce that

$$
\lim _{\tau \rightarrow t}\|u(t)-u(\tau)\|_{H^{2}(\omega)} \leq 0
$$

In conclusion, $u \in \mathcal{C}\left([0, T], H^{2}(\omega)\right)$.

Let us close this section by introducing an appropriate variational formulation for the forward problem (1)-(5) which is important for our shape sensitivity analysis:

$$
\text { Find } u \in X_{0}: \quad \int_{0}^{T} \int_{\mathcal{D}} R \nabla u \cdot \nabla \psi-\kappa u_{t} \psi_{t}+\eta u_{t} \psi d x d t=\int_{0}^{T} \int_{\mathcal{D}} f \psi d x d t \quad \forall \psi \in X_{T} .
$$

Theorem 2. Let Assumption 1 hold and $f \in W^{1,1}\left((0, T), L^{2}(\mathcal{D})\right)$. Then, the variational problem (29) admits a unique solution $u \in X_{0}$, which coincides with the strong solution to (1)-(5). In particular, the unique solution to $\left[29\right.$ enjoys the regularity property $u \in X_{0} \cap \mathcal{C}\left([0, T], H_{\Gamma}^{1}(\mathcal{D})\right) \cap \mathcal{C}^{2}\left([0, T], L^{2}(\mathcal{D})\right)$.

Proof. Uniqueness: Suppose that $u^{(1)}, u^{(2)} \in X_{0}$ are solutions to (29). By definition, the difference $d:=$

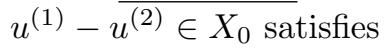

$$
\int_{0}^{T} \int_{\mathcal{D}} R \nabla d \cdot \nabla \psi-\kappa d_{t} \psi_{t}+\eta d_{t} \psi d x d t=0 \quad \forall \psi \in X_{T} .
$$

Testing the above variational equality with $\psi:=\mu \varphi$ for $\mu \in \mathcal{C}_{0}^{\infty}(0, T)$ and $\varphi \in H_{\Gamma}^{1}(\mathcal{D})$ yields

$$
-\int_{0}^{T} \mu^{\prime}(t) \int_{\mathcal{D}} \kappa d_{t}(t) \varphi d x d t+\int_{0}^{T} \mu(t) \int_{\mathcal{D}} R \nabla d(t) \cdot \nabla \varphi+\eta d_{t}(t) \varphi d x d t=0 \quad \forall \mu \in \mathcal{C}_{0}^{\infty}(0, T),
$$

from which it follows by the definition of the weak (time) derivative that

$$
\frac{d}{d t} \int_{\mathcal{D}} \kappa d_{t}(t) \varphi d x+\int_{\mathcal{D}} R \nabla d(t) \cdot \nabla \varphi+\eta d_{t}(t) \varphi d x=0 \quad \text { for all } \varphi \in H_{\Gamma}^{1}(\mathcal{D}) \text { and a.e. } t \in(0, T) .
$$


Using the fact that $\int_{\mathcal{D}} \eta d_{t}(t) d(t) d x=\frac{1}{2} \frac{d}{d t}\left\|\eta^{\frac{1}{2}} d(t)\right\|_{L^{2}(\mathcal{D})}^{2}$ and $d(0)=0$, we obtain by setting $\varphi=d(t)$ and integrating the resulting equality over the time interval $[0, \tau]$ for any $0 \leq \tau \leq T$ that

$$
\int_{\mathcal{D}} \kappa d_{t}(\tau) d(\tau) d x+\int_{0}^{\tau} \int_{\mathcal{D}} R \nabla d(t) \cdot \nabla d(t) d x d t+\frac{1}{2}\left\|\eta^{\frac{1}{2}} d(\tau)\right\|_{L^{2}(\mathcal{D})}^{2}=0 .
$$

Thus, it follows that

$$
\frac{d}{d t}\left\|\kappa^{\frac{1}{2}} d(t)\right\|_{L^{2}(\mathcal{D})}^{2}=\int_{\mathcal{D}} \kappa d_{t}(t) d(t) d x \leq 0 \quad \text { for a.e. } t \in(0, T) \underbrace{\Longrightarrow}_{d(0)=0} d \equiv 0 .
$$

Existence: As $f \in W^{1,1}\left((0, T), L^{2}(\mathcal{D})\right)$, Corollary 1 yields that the mild solution to (1)-(5) enjoys the regularity property $u \in \mathcal{C}\left([0, T], H_{\Gamma}^{1}(\mathcal{D})\right) \cap \mathcal{C}^{2}\left([0, T], L^{2}(\mathcal{D})\right)$ and satisfies $[23)$, i.e., it is the strong solution to (1)-(5). It is straightforward to see that $u$ is a solution to the variational problem (29).

\section{Shape optimization Setting}

In this section we describe a general shape optimization framework to find an approximation solution of the inverse problem. The key tool to perform the sensitivity analysis of the problem is the notion of shape derivative, which we shall briefly recall in the following. Let us first denote the set of admissible shapes by

$$
\mathbb{P}(\mathcal{D}):=\{\Omega \subset \mathcal{D} \mid \Omega \text { open, } \partial \Omega \cap \partial \mathcal{D}=\emptyset\}
$$

and introduce $F: \mathbb{R} \times \mathbb{R}^{n} \times[0, T] \rightarrow \mathbb{R}$ for the shape functional as follows:

$$
F(u, x, t)=\frac{1}{2} \sum_{\rho=1}^{N_{\rho}} w_{\rho}(x)\left(u-d_{\rho}(t)\right)^{2}, \quad N_{\rho} \in \mathbb{N},
$$

for given mappings $d_{\rho}:(0, T) \rightarrow \mathbb{R}$ and $w_{\rho}: \mathcal{D} \rightarrow \mathbb{R}$. The required mathematical assumptions for this function and all other data involved in the shape optimization problem are summarized as follows:

Assumption 3. Let $\Omega \in \mathbb{P}(\mathcal{D})$. The material parameters $\kappa$ and $R$ are assumed to be piecewise constant:

$$
\kappa=\kappa_{\Omega}=\kappa_{0} \chi_{\Omega}+\kappa_{1} \chi_{\mathcal{D} \backslash \Omega} \quad \text { and } \quad R=R_{\Omega}=R_{0} \chi_{\Omega}+R_{1} \chi_{\mathcal{D} \backslash \Omega}
$$

with positive real constants $\kappa_{0}, \kappa_{1}>0$ and symmetric and positive definite matrices $R_{0}, R_{1} \in \mathbb{R}^{n \times n}$. Furthermore, suppose that $\eta \in L^{\infty}(\mathcal{D})$ is nonnegative, and $f \in W^{1,1}\left((0, T), L^{2}(\mathcal{D})\right)$ is given. Concerning (30), we assume that $d_{\rho} \in W^{1,1}((0, T), \mathbb{R})$ and $w_{\rho} \in \mathcal{C}^{2}(\overline{\mathcal{D}})$ for all $\rho=1, \ldots, N_{\rho}$.

Under Assumption 3, our focus is set on the following shape optimization problem:

$$
\min \mathcal{J}(u, \Omega):=\int_{0}^{T} \int_{\mathcal{D}} F(u(x, t), x, t) d x d t
$$

subject to $\Omega \in \mathbb{P}(\mathcal{D})$ and 29 .

In view of $(30), \mathcal{J}$ is a general misfit functional where $d_{\rho}$ represents the observed data at a receiver indexed by $\rho$. The precise meaning of $w_{\rho}$ and $d_{\rho}$ in the context of FWI is described in Section 6. Denoting by $u(\Omega) \in X_{0} \cap \mathcal{C}\left([0, T], H_{\Gamma}^{1}(\mathcal{D})\right) \cap \mathcal{C}^{2}\left([0, T], L^{2}(\mathcal{D})\right)$ the unique solution to $\left[29\right.$ associated with $\kappa=\kappa_{\Omega}$ and $R=R_{\Omega}$ (see Assumption 3), the minimization problem (31) can be equivalently reformulated as

$$
\min _{\Omega \in \mathbb{P}(\mathcal{D})} J(\Omega):=\mathcal{J}(u(\Omega), \Omega) .
$$

We now recall some basics regarding the sensitivity analysis of shape functionals. For given $k \geq 0$ and $0 \leq \alpha \leq 1$, we define

$$
\mathcal{C}_{c}^{k, \alpha}\left(\mathcal{D}, \mathbb{R}^{n}\right):=\left\{\theta \in \mathcal{C}^{k, \alpha}\left(\mathcal{D}, \mathbb{R}^{n}\right) \mid \theta \text { has compact support in } \mathcal{D}\right\} .
$$

Let $\theta \in \mathcal{C}_{c}^{0,1}\left(\mathcal{D}, \mathbb{R}^{n}\right)$ and consider the associated flow $\Phi_{s}\left(x_{0}\right)=x\left(s, x_{0}\right)$ defined by the solution to the ordinary differential equation

$$
\frac{\mathrm{d}}{\mathrm{d} s} x\left(s, x_{0}\right)=\theta\left(x\left(s, x_{0}\right)\right) \quad \text { for } s \in\left[0, s_{0}\right], \quad x\left(0, x_{0}\right)=x_{0} \in \mathcal{D},
$$

for some given $s_{0}>0$. It is well-known (see [42, p. 50]) that (33) admits a unique solution for a sufficiently small $s_{0}>0$. Note that $\Phi_{s}(\mathcal{D})=\mathcal{D}$ since $\theta$ has compact support in $\mathcal{D}$. For $\Omega \in \mathbb{P}(\mathcal{D})$, we introduce the 
parameterized family of domains $\Omega_{s}:=\Phi_{s}(\Omega)$, for all $s \in\left[0, s_{0}\right]$. Let us now recall the definition of the shape derivative used in this paper.

Definition 1 (Shape derivative). Let $K: \mathbb{P}(\mathcal{D}) \rightarrow \mathbb{R}$ be a shape functional. The Eulerian semiderivative of $K$ at $\Omega \in \mathbb{P}(\mathcal{D})$ in direction $\theta \in \mathcal{C}_{c}^{0,1}\left(\mathcal{D}, \mathbb{R}^{n}\right)$ is defined as the limit, if it exists,

$$
d K(\Omega)(\theta):=\lim _{s \searrow 0} \frac{K\left(\Omega_{s}\right)-K(\Omega)}{s} .
$$

Moreover, $K$ is said to be shape differentiable at $\Omega$ if it has a Eulerian semiderivative at $\Omega$ for all $\theta \in$ $\mathcal{C}_{c}^{0,1}\left(\mathcal{D}, \mathbb{R}^{n}\right)$, and the mapping

$$
d K(\Omega): \mathcal{C}_{c}^{0,1}\left(\mathcal{D}, \mathbb{R}^{n}\right) \rightarrow \mathbb{R}, \quad \theta \mapsto d K(\Omega)(\theta),
$$

is linear and continuous. In this case $d K(\Omega)(\theta)$ is called the shape derivative of $K$ at $\Omega$ in direction $\theta$.

\section{Averaged adjoint method}

In this section we describe the averaged adjoint method introduced in 44 to establish the shape derivative of the reduced cost functional $J(\Omega)=\mathcal{J}(u(\Omega), \Omega)$. The notations are adapted to the particular setting of our problem, and we refer to [30] or 44] for a presentation of the method in the general case.

In all what follows, let Assumption 3 hold. Furthermore, let $\theta \in \mathcal{C}_{c}^{0,1}\left(\mathcal{D}, \mathbb{R}^{n}\right)$ with the associated flow $\Phi_{s}: \mathcal{D} \rightarrow \mathcal{D}$ and $\Omega_{s}=\Phi_{s}(\Omega)$ as in Section 3 Furthermore, we write $u_{s}=u\left(\Omega_{s}\right)$ for the unique solution to 29) associated with $\kappa=\kappa_{\Omega_{s}}=\kappa_{0} \chi_{\Omega_{s}}+\kappa_{1} \chi_{\mathcal{D} \backslash \Omega_{s}}$ and $R=R_{\Omega_{s}}=R_{0} \chi_{\Omega_{s}}+R_{1} \chi_{\mathcal{D} \backslash \Omega_{s}}$.

The averaged adjoint method relies on the use of the Lagrangian $\mathcal{L}: \mathbb{P}(\mathcal{D}) \times X_{0} \times X_{T} \rightarrow \mathbb{R}$ associated with the minimization problem $(32)$ as follows

$$
\mathcal{L}(\Omega, \varphi, \psi):=\int_{0}^{T} \int_{\mathcal{D}} F(\varphi(x, t), x, t)+R \nabla \varphi \cdot \nabla \psi-\kappa \varphi_{t} \psi_{t}+\eta \varphi_{t} \psi-f \psi d x d t .
$$

By definition, it holds that

$$
J\left(\Omega_{s}\right)=\mathcal{L}\left(\Omega_{s}, u_{s}, \psi\right) \quad \forall \psi \in X_{T} .
$$

Thus, we can compute the shape derivative using

$$
d J(\Omega)(\theta)=\left.\frac{\mathrm{d}}{\mathrm{d} s} \mathcal{L}\left(\Omega_{s}, u_{s}, \psi\right)\right|_{s=0} \quad \forall \psi \in X_{T} .
$$

We will see shortly that the above calculation can be significantly simplified by choosing a particular $\psi$. In order to differentiate $\mathcal{L}\left(\Omega_{s}, \varphi, \psi\right)$ with respect to $s$, the change of coordinates $x \mapsto \Phi_{s}(x)$ is used in (34). In the process the compositions $\varphi \circ \Phi_{s}$ and $\psi \circ \Phi_{s}$ appear, which creates differentiability issues. To compensate this effect, one considers the reparameterized Lagrangian $\mathcal{L}\left(\Omega_{s}, \Psi_{s}^{-1}(\varphi), \Psi_{s}^{-1}(\psi)\right)$ where the pullback $\Psi_{s}$ is defined by $\Psi_{s}(\psi)=\psi \circ \Phi_{s}$. It can be checked that $\Psi_{s}: X_{0} \rightarrow X_{0}$ and $\Psi_{s}: X_{T} \rightarrow X_{T}$ are bijections; see [49, Theorem 2.2.2, p. 52]. Thus we introduce the so-called parameterized shape-Lagrangian $G:\left[0, s_{0}\right] \times X_{0} \times X_{T} \rightarrow \mathbb{R}$ as

$$
G(s, \varphi, \psi):=\mathcal{L}\left(\Omega_{s}, \varphi \circ \Phi_{s}^{-1}, \psi \circ \Phi_{s}^{-1}\right) .
$$

Denote by $u^{s}:=u_{s} \circ \Phi_{s} \in X_{0}$ and by $d_{\psi} G\left(s, u^{s}, 0 ; \hat{\psi}\right)$ the directional derivative of $G$ with respect to $\psi$ in direction $\hat{\psi}$ at $\left(s, u^{s}, 0\right)$, it can be checked, using the change of coordinates $x \mapsto \Phi_{s}(x)$, that the following equation for $u^{s}$ :

$$
d_{\psi} G\left(s, u^{s}, 0 ; \hat{\psi}\right)=0 \quad \forall \hat{\psi} \in X_{T}
$$

is equivalent to the state equation (29) with $\kappa=\kappa_{\Omega_{s}}$ and $R=R_{\Omega_{s}}$; see 61] for an explicit expression of equation (37).

For the convenience of the reader, we provide now the main result of the averaged adjoint method, adapted to our case. A proof can be found in [44] or [30. Theorem 2.1]. The main idea of this result is to show that the shape derivative can be computed via the partial derivative of $G$ with respect to $s$ under the use of the averaged adjoint state. Observe that $u=u^{0}$ so we will use the notation $u$ in what follows, and we also use the notation $p$ instead of $p^{0}$ for the adjoint.

Theorem 3 (Averaged adjoint method). Suppose that there exists $s_{0}>0$ such that for every $(s, \psi) \in$ $\left[0, s_{0}\right] \times X_{T}$ the following conditions are satisfied: 
(H1) the mapping $[0,1] \ni \zeta \mapsto G\left(s, \zeta u^{s}+(1-\zeta) u, \psi\right)$ is absolutely continuous;

(H2) the mapping $[0,1] \ni \zeta \mapsto d_{\varphi} G\left(s, \zeta u^{s}+(1-\zeta) u, \psi ; \hat{\varphi}\right)$ belongs to $L^{1}(0,1)$ for all $\hat{\varphi} \in X_{0}$;

(H3) there exists a unique solution $p^{s} \in X_{T}$ to the averaged adjoint equation

$$
\int_{0}^{1} d_{\varphi} G\left(s, \zeta u^{s}+(1-\zeta) u, p^{s} ; \hat{\varphi}\right) d \zeta=0 \quad \text { for all } \hat{\varphi} \in X_{0}
$$

(H4) we have

$$
\lim _{s \searrow 0} \frac{G\left(s, u, p^{s}\right)-G\left(0, u, p^{s}\right)}{s}=\partial_{s} G(0, u, p) .
$$

Then $J$ is shape differentiable with

$$
d J(\Omega)(\theta)=\partial_{s} G(0, u, p) .
$$

In view of (39), the shape derivative depends on the adjoint state $p \in X_{T}$. Taking $s=0$ in (38), the adjoint equation reads as:

$$
\text { Find } p \in X_{T}: \quad \partial_{\varphi} G(0, u, p ; \hat{\varphi})=0 \quad \forall \hat{\varphi} \in X_{0},
$$

where $u \in X_{0} \cap \mathcal{C}\left([0, T], H_{\Gamma}^{1}(\mathcal{D})\right) \cap \mathcal{C}^{2}\left([0, T], L^{2}(\mathcal{D})\right)$ is the unique solution to 29 with $\kappa=\kappa_{\Omega}$ and $R=R_{\Omega}$. Using $\partial_{u} F(u, x, t)=\sum_{\rho=1}^{N_{\rho}} w_{\rho}(x)\left(u-d_{\rho}(t)\right)$ and (34), the variational problem (40) is equivalent to

$$
\int_{0}^{T} \int_{\mathcal{D}} R \nabla \hat{\varphi} \cdot \nabla p-\kappa \hat{\varphi}_{t} p_{t}+\eta \hat{\varphi}_{t} p d x d t=-\int_{0}^{T} \int_{\mathcal{D}} \partial_{u} F(u(x, t), x, t) \hat{\varphi} d x d t \quad \forall \hat{\varphi} \in X_{0} .
$$

Our goal now is to show that the adjoint state satisfies a backwards wave equation with terminal conditions and determine the strong form of the adjoint equation. To this aim, we consider the following auxiliary problem

$$
\begin{aligned}
\kappa q_{t t}-\operatorname{div}(R \nabla q)+\eta q_{t} & =-\partial_{u} F(u(T-t), \cdot, T-t) \text { in } \mathcal{D} \times[0, T], \\
q(0) & =0 \text { in } \mathcal{D}, \\
q_{t}(0) & =0 \text { in } \mathcal{D}, \\
q & =0 \text { on } \Gamma \times[0, T], \\
R \nabla q \cdot n & =0 \text { on } \Gamma_{n} \times[0, T] .
\end{aligned}
$$

Since $u \in \mathcal{C}^{2}\left([0, T], L^{2}(\mathcal{D})\right)$, Assumption 3 ensures that $t \mapsto \partial_{u} F(u(T-t), \cdot, T-t)$ is of class $W^{1,1}\left((0, T), L^{2}(\mathcal{D})\right)$ such that Corollary 1 yields the existence of a unique strong solution $q \in \mathcal{C}\left([0, T], H_{\Gamma}^{1}(\mathcal{D})\right) \cap \mathcal{C}^{2}\left([0, T], L^{2}(\mathcal{D})\right)$ of (42)-46). According to Theorem 2, the strong solution to 42 - 46 is exactly the unique solution to the variational problem

$$
\int_{0}^{T} \int_{\mathcal{D}} R \nabla q \cdot \nabla \psi-\kappa q_{t} \psi_{t}+\eta q_{t} \psi d x d t=\int_{0}^{T} \int_{\mathcal{D}}-\partial_{u} F(u(x, T-t), x, T-t) \psi d x d t \quad \forall \psi \in X_{T} .
$$

Now, introducing $\hat{p}(t):=q(T-t)$ we obtain

$$
\kappa \hat{p}_{t t}(t)-\operatorname{div}(R \nabla \hat{p}(t))-\eta \hat{p}_{t}(t)=\partial_{u} F(u(t), \cdot, t) \quad \forall t \in[0, T],
$$

and $\hat{p} \in X_{T} \cap \mathcal{C}\left([0, T], H_{\Gamma}^{1}(\mathcal{D})\right) \cap \mathcal{C}^{2}\left([0, T], L^{2}(\mathcal{D})\right)$. Defining the test function $\hat{\varphi} \in X_{0}$ by $\hat{\varphi}(t):=\psi(T-t)$, proceeding with the change of variables $t \mapsto T-t$ in (47) and integrating by parts in time the term depending on $\eta q_{t} \psi$, we obtain the same equation as (41) for $\hat{p}$, also using the fact that $R$ is symmetric. This shows that $\hat{p} \in X_{T} \cap \mathcal{C}\left([0, T], H_{\Gamma}^{1}(\mathcal{D})\right) \cap \mathcal{C}^{2}\left([0, T], L^{2}(\mathcal{D})\right)$ is the unique solution to 41 where the uniqueness follows the same argument as in Theorem 2, In conclusion, we have shown the following result:

Theorem 4. Let Assumption 3 hold, and let $u \in X_{0} \cap \mathcal{C}\left([0, T], H_{\Gamma}^{1}(\mathcal{D})\right) \cap \mathcal{C}^{2}\left([0, T], L^{2}(\mathcal{D})\right)$ denote the unique solution to 29 . Then, the variational problem

$$
\int_{0}^{T} \int_{\mathcal{D}} R \nabla \hat{\varphi} \cdot \nabla p-\kappa \hat{\varphi}_{t} p_{t}+\eta \hat{\varphi}_{t} p=-\int_{0}^{T} \int_{\mathcal{D}} \partial_{u} F(u(x, t), x, t) \hat{\varphi} \quad \forall \hat{\varphi} \in X_{0}
$$


admits a unique solution $p \in X_{T} \cap \mathcal{C}\left([0, T], H_{\Gamma}^{1}(\mathcal{D})\right) \cap \mathcal{C}^{2}\left([0, T], L^{2}(\mathcal{D})\right)$ satisfying the following backwards wave equations with terminal conditions:

$$
\begin{aligned}
\kappa p_{t t}(t)-\operatorname{div}(R \nabla p(t))-\eta p_{t}(t) & =-\partial_{u} F(u(t), \cdot, t) \text { in } \mathcal{D} \times(0, T), \\
p(T) & =0 \text { in } \Omega, \\
p_{t}(T) & =0 \text { in } \Omega, \\
u & =0 \text { on } \Gamma \times(0, T), \\
R \nabla u \cdot n & =0 \text { on } \Gamma_{n} \times(0, T) .
\end{aligned}
$$

\section{Shape DiffEREnTIABILITY AND Shape DERIVATIVE}

Applying the averaged adjoint method presented in Section 4 , we prove the shape differentiability and provide the expression of the distributed shape derivative of the cost functional using a tensorial representation, in the spirit of 29,30 .

Theorem 5. Let Assumption 3 hold with $\eta \in \mathcal{C}^{1}(\mathcal{D})$ and $f \in W^{1,1}\left((0, T), H^{1}(\mathcal{D})\right)$. Suppose that $\theta \in$ $\mathcal{C}_{c}^{0,1}\left(\mathcal{D}, \mathbb{R}^{n}\right)$. Furthermore, let $u \in X_{0} \cap \mathcal{C}\left([0, T], H_{\Gamma}^{1}(\mathcal{D})\right) \cap \mathcal{C}^{2}\left([0, T], L^{2}(\mathcal{D})\right)$ and $p \in X_{T} \cap \mathcal{C}\left([0, T], H_{\Gamma}^{1}(\mathcal{D})\right) \cap$ $\mathcal{C}^{2}\left([0, T], L^{2}(\mathcal{D})\right)$ denote, respectively, the unique solutions to (29) and (48). Then, the shape derivative of $J$ at $\Omega$ in direction $\theta$ is given by

$$
d J(\Omega)(\theta)=\int_{\mathcal{D}} \mathbf{S}_{1}: D \theta+\mathbf{S}_{0} \cdot \theta,
$$

with $\mathbf{S}_{1} \in L^{1}\left(\mathcal{D}, \mathbb{R}^{n \times n}\right)$ and $\mathbf{S}_{0} \in L^{1}\left(\mathcal{D}, \mathbb{R}^{n}\right)$ defined by

$$
\begin{aligned}
& \mathbf{S}_{1}=\left[\int_{0}^{T} F(u(t), \cdot, t)-\kappa u_{t} p_{t}+R \nabla u \cdot \nabla p+\eta u_{t} p-f p d t\right] I_{n}-\int_{0}^{T} \nabla u \otimes R \nabla p+\nabla p \otimes R \nabla u d t, \\
& \mathbf{S}_{0}=\int_{0}^{T} \nabla_{x} F(u(t), \cdot, t)+p u_{t} \nabla \eta-p \nabla f d t,
\end{aligned}
$$

where $I_{n} \in \mathbb{R}^{n \times n}$ is the identity matrix and $\nabla_{x} F(u, x, t)=\frac{1}{2} \sum_{\rho=1}^{N_{\rho}} \nabla w_{\rho}(x)\left(u-d_{\rho}(t)\right)^{2}$.

Proof. We check that the assumptions of Theorem 3 are satisfied. Before computing the shape-Lagrangian $G$ defined in (36), a few remarks are in order. Introducing $\kappa_{s}=\kappa_{\Omega_{s}}=\kappa_{0} \chi_{\Omega_{s}}+\kappa_{1} \chi_{\mathcal{D} \backslash \Omega_{s}}$, we have

$$
\kappa_{\Omega_{s}} \circ \Phi_{s}=\kappa_{0} \chi_{\Omega_{s}} \circ \Phi_{s}+\kappa_{1} \chi_{\mathcal{D} \backslash \Omega_{s}} \circ \Phi_{s}=\kappa_{0} \chi_{\Omega}+\kappa_{1} \chi_{\mathcal{D} \backslash \Omega}=\kappa_{\Omega},
$$

and in a similar way $R_{\Omega_{s}} \circ \Phi_{s}=R_{\Omega}$. We also have $\partial_{t}\left(\varphi \circ \Phi_{s}^{-1}\right)=\partial_{t} \varphi \circ \Phi_{s}^{-1}$ since $\Phi_{s}$ is independent of $t$.

Applying definitions (36) and (34) as well as $\Phi_{s}(\mathcal{D})=\mathcal{D}$, and proceeding with the change of coordinates $x \mapsto \Phi_{s}(x)$ in the integrals, we get the shape-Lagrangian $G:\left[0, s_{0}\right] \times X_{0} \times X_{T} \rightarrow \mathbb{R}$ as

$$
\begin{aligned}
G(s, \varphi, \psi)= & \int_{0}^{T} \int_{\mathcal{D}} F\left(\varphi(x, t), \Phi_{s}(x), t\right) \xi(s) \\
& +\int_{0}^{T} \int_{\mathcal{D}} A(s) \nabla \varphi \cdot \nabla \psi-\kappa \varphi_{t} \psi_{t} \xi(s)+\eta_{s} \varphi_{t} \psi \xi(s)-f_{s} \psi \xi(s),
\end{aligned}
$$

with $\xi(s):=\left|\operatorname{det}\left(D \Phi_{s}\right)\right|, A(s):=\xi(s)\left(D \Phi_{s}\right)^{-1} R\left(D \Phi_{s}\right)^{-\top}, f_{s}:=f \circ \Phi_{s}$, and $\eta_{s}=\eta \circ \Phi_{s}$. For $s$ sufficiently small we have $\xi(s)=\operatorname{det}\left(D \Phi_{s}\right)>0$. The following asymptotic expansions hold (see [42, Lemma 2.31]):

$$
\xi(s)=1+s \operatorname{div}(\theta)+o(s), D \Phi_{s}=I+s D \theta+o(s), D \Phi_{s}^{-1}=I-s D \theta+o(s),
$$

with $o(s) / s \rightarrow 0$ as $s \rightarrow 0$ with respect to $\|\cdot\|_{\mathcal{C}(\Omega)}$ and $\|\cdot\|_{\mathcal{C}\left(\Omega, \mathbb{R}^{3 \times 3}\right)}$, respectively. Note that $A(s)$ is definite positive due to $(59)$. The asymptotic expansions $(59)$ imply that there exists a constant $C>0$ only dependent on $\theta$ such that

$$
\|\xi(s)\|_{L^{\infty}(\Omega)}+\left\|D \Phi_{s}\right\|_{L^{\infty}\left(\Omega, \mathbb{R}^{3 \times 3}\right)}+\left\|D \Phi_{s}^{-1}\right\|_{L^{\infty}\left(\Omega, \mathbb{R}^{3 \times 3}\right)} \leq 1+C s_{0} .
$$

Using (58), we obtain the following explicit expression for the equation (37) of $u^{s}:=u_{s} \circ \Phi_{s}$ :

$$
\int_{0}^{T} \int_{\mathcal{D}} A(s) \nabla u^{s} \cdot \nabla \hat{\psi}-\kappa \partial_{t} u^{s} \partial_{t} \hat{\psi} \xi(s)+\eta_{s} \partial_{t} u^{s} \hat{\psi} \xi(s)=\int_{0}^{T} \int_{\mathcal{D}} f_{s} \hat{\psi} \xi(s) \text { for all } \hat{\psi} \in X_{T} .
$$


We first check condition (H1) of Theorem 3 . We compute

$$
\begin{aligned}
\frac{\mathrm{d}}{\mathrm{d} \zeta} G\left(s, \zeta u^{s}+(1-\zeta) u, \psi\right) & =\int_{0}^{T} \int_{\mathcal{D}} \partial_{u} F\left(\zeta u^{s}+(1-\zeta) u, \Phi_{s}(x), t\right)\left(u^{s}-u\right) \xi(s) \\
& +\int_{0}^{T} \int_{\mathcal{D}} A(s) \nabla\left(u^{s}-u\right) \cdot \nabla \psi-\kappa \partial_{t}\left(u^{s}-u\right) \psi_{t} \xi(s)+\eta_{s} \partial_{t}\left(u^{s}-u\right) \psi \xi(s),
\end{aligned}
$$

where

$$
\partial_{u} F(u, x, t)=\sum_{\rho=1}^{N_{\rho}} w_{\rho}(x)\left(u-d_{\rho}(t)\right) .
$$

Using Assumption 3. $\zeta \in[0,1], 600,62), \psi \in X_{T}$, and $u^{s} \in \mathcal{C}\left([0, T], H_{\Gamma}^{1}(\mathcal{D})\right) \cap \mathcal{C}^{1}\left([0, T], L^{2}(\mathcal{D})\right)$ we get

$$
\left|\frac{\mathrm{d}}{\mathrm{d} \zeta} G\left(s, \zeta u^{s}+(1-\zeta) u, \psi\right)\right|=C_{1}+C \int_{0}^{T} \int_{\mathcal{D}}\left(1+\left|u^{s}\right|+|u|\right)\left|u^{s}-u\right| \leq C_{2} .
$$

This shows that the mapping $[0,1] \ni \zeta \mapsto G\left(s, \zeta u^{s}+(1-\zeta) u, \psi\right)$ is Lipschitz and therefore absolutely continuous, hence condition (H1) is satisfied.

Now we check condition (H2) of Theorem 3 . We have, using Assumption 3 .

$$
\begin{aligned}
\int_{0}^{1}\left|d_{\varphi} G\left(s, \zeta u^{s}+(1-\zeta) u, \psi ; \hat{\varphi}\right)\right| d \zeta \leq & \underbrace{\int_{0}^{T} \int_{\mathcal{D}}\left|A(s) \nabla \hat{\varphi} \cdot \nabla \psi-\kappa \hat{\varphi}_{t} \psi_{t} \xi(s)+\eta_{s} \hat{\varphi}_{t} \psi \xi(s)\right|}_{\leq C_{1} \text { due to }\left[59,, \psi \in X_{T} \text { and } \hat{\varphi} \in X_{0}\right.} \\
& +\int_{0}^{T} \int_{\mathcal{D}}\left(\int_{0}^{1}\left|\partial_{u} F\left(\zeta u^{s}+(1-\zeta) u, \Phi_{s}(x), t\right) \xi(s) \hat{\varphi}\right| d \zeta\right) . \\
\leq & C_{1}+\underbrace{C \underbrace{\int_{0}^{T} \int_{\mathcal{D}}|\xi(s) \hat{\varphi}|\left(\int_{0}^{1} 1+\left|\zeta u^{s}+(1-\zeta) u\right| d \zeta\right)}_{0}}_{\leq C_{2} \text { due to } u^{s} \in X_{0} \text { and }} .
\end{aligned}
$$

where we have used 62 and Assumption 3 . This shows that condition (H2) is satisfied.

Then it is easy to check that the averaged adjoint equation (38) for $p^{s} \in X_{T}$ is given by

$$
\begin{aligned}
& \int_{0}^{T} \int_{\mathcal{D}} A(s) \nabla \hat{\varphi} \cdot \nabla p^{s}-\kappa \hat{\varphi}_{t} p_{t}^{s} \xi(s)+\eta \hat{\varphi}_{t} p^{s} \xi(s) d x d t \\
& =-\int_{0}^{1} \int_{0}^{T} \int_{\mathcal{D}} \hat{\varphi} \partial_{u} F\left(\zeta u^{s}+(1-\zeta) u, \Phi_{s}(x), t\right) \xi(s) d x d t d \zeta, \text { for all } \hat{\varphi} \in X_{0} .
\end{aligned}
$$

Introducing an auxiliary function $q^{s}(t):=p^{s}(T-t)$ and test functions $\tilde{\varphi}(t):=\hat{\varphi}(T-t)$, we have that $q^{s} \in X_{0}$ and $\tilde{\varphi} \in X_{T}$. Using Fubini's theorem, a change of variables $t \mapsto T-t$ in $(63)$, an integration by part with respect to $t$ for the term $\eta \tilde{\varphi}_{t} q^{s} \xi(s)$ and the fact that $A(s)$ is symmetric, one obtains the following equation for $q^{s}$ :

$$
\int_{0}^{T} \int_{\mathcal{D}} A(s) \nabla q^{s} \cdot \nabla \tilde{\varphi}-\kappa \tilde{\varphi}_{t} q_{t}^{s} \xi(s)+\eta \tilde{\varphi} q_{t}^{s} \xi(s) d x d t=-\int_{0}^{T} \int_{\mathcal{D}} \hat{f}_{s} \tilde{\varphi} d x d t, \text { for all } \tilde{\varphi} \in X_{T}
$$

where

$$
\hat{f}_{s}(x, t):=\xi(s) \int_{0}^{1} \partial_{u} F\left(\zeta u^{s}(x, T-t)+(1-\zeta) u(x, T-t), \Phi_{s}(x), T-t\right) d \zeta .
$$

Using 62 we get

$$
\begin{aligned}
\hat{f}_{s}(x, t) & =\xi(s) \sum_{\rho=1}^{N_{\rho}} w_{\rho}\left(\Phi_{s}(x)\right)\left(\frac{1}{2} u^{s}(x, T-t)+\frac{1}{2} u(x, T-t)-d_{\rho}(T-t)\right), \\
\partial_{t} \hat{f}_{s}(x, t) & =-\xi(s) \sum_{\rho=1}^{N_{\rho}} w_{\rho}\left(\Phi_{s}(x)\right)\left(\frac{1}{2} \partial_{t} u^{s}(x, T-t)+\frac{1}{2} \partial_{t} u(x, T-t)-d_{\rho}^{\prime}(T-t)\right) .
\end{aligned}
$$


Using Assumption 3 and 62 we obtain the estimates

$$
\begin{gathered}
\int_{\mathcal{D}}\left(\int_{0}^{T}\left|\hat{f}_{s}\right| d t\right)^{2} d x \leq C_{0} \sum_{\rho=1}^{N_{\rho}}\left\|d_{\rho}\right\|_{L^{1}}^{2}+C_{1} \int_{\mathcal{D}}\left(\int_{0}^{T}\left|u^{s}(x, T-t)+u(x, T-t)\right| d t\right)^{2} d x<\infty, \\
\int_{\mathcal{D}}\left(\int_{0}^{T}\left|\partial_{t} \hat{f}_{s}\right| d t\right)^{2} d x \leq C_{0} \sum_{\rho=1}^{N_{\rho}}\left\|d_{\rho}\right\|_{W^{1,1}}^{2}+C_{1} \int_{\mathcal{D}}\left(\int_{0}^{T}\left|\partial_{t} u^{s}(x, T-t)+\partial_{t} u(x, T-t)\right| d t\right)^{2} d x<\infty,
\end{gathered}
$$

where we have used 600 and the fact that $u^{s} \in \mathcal{C}\left([0, T], H_{\Gamma}^{1}(\mathcal{D})\right) \cap \mathcal{C}^{1}\left([0, T], L^{2}(\mathcal{D})\right)$. This shows that $\hat{f}_{s} \in W^{1,1}\left((0, T), L^{2}(\mathcal{D})\right)$. Thus, we can apply Corollary 1 to equation 64 with $A(s)$ instead of $R, \kappa \xi(s)$ instead of $\kappa$, and $\eta \xi(s)$ instead of $\eta$, since $A(s), \kappa \xi(s)$ and $\eta \xi(s)$ satisfy Assumption 1 . This shows that the equation for $q^{s}$ admits a unique mild solution $q^{s} \in \mathcal{C}\left([0, T], H_{\Gamma}^{1}(\mathcal{D})\right) \cap \mathcal{C}^{1}\left([0, T], L^{2}(\mathcal{D})\right)$. Consequently, (63) also admits a unique mild solution $p^{s} \in \mathcal{C}\left([0, T], H_{\Gamma}^{1}(\mathcal{D})\right) \cap \mathcal{C}^{1}\left([0, T], L^{2}(\mathcal{D})\right)$. This shows that condition $(\mathrm{H} 3)$ of Theorem 3 is satisfied.

Now we verify Assumption (H4) of Theorem 3 . First of all, we have

$$
\begin{aligned}
\frac{G\left(s, u, p^{s}\right)-G\left(0, u, p^{s}\right)}{s} & =\int_{0}^{T} \int_{\mathcal{D}} \frac{F\left(u, \Phi_{s}(x), t\right) \xi(s)-F(u, x, t)}{s} \\
& +\int_{0}^{T} \int_{\mathcal{D}} \frac{A(s)-I_{n}}{s} \nabla u \cdot \nabla p^{s}-\kappa u_{t} p_{t}^{s} \frac{\xi(s)-1}{s} \\
& +\int_{0}^{T} \int_{\mathcal{D}} \frac{\eta_{s} \xi(s)-\eta}{s} u_{t} p^{s}-\frac{f_{s} \xi(s)-f}{s} p^{s} .
\end{aligned}
$$

To calculate the limit $s \rightarrow 0$ of the above expression, we first need a uniform estimate on $p^{s}$. In view of (9), [65), using Assumption 3 and the fact that $\kappa \xi(s)$ is uniformly bounded, we also have

$$
\begin{aligned}
C_{1}\left\|q^{s}\right\|_{X}^{2} & \leq \int_{0}^{T} \int_{\mathcal{D}} \kappa \xi(s)\left(q_{t}^{s}\right)^{2}(t) d x+\int_{\mathcal{D}} A(s) \nabla q^{s}(t) \cdot \nabla q^{s}(t) d x \\
& \underbrace{\leq}_{\sqrt[9]{9}} \int_{0}^{T}\left(\int_{0}^{t}\left\|(\kappa \xi(s))^{\frac{1}{2}} \hat{f}_{s}(x, \sigma)\right\|_{L^{2}(\mathcal{D})} d \sigma\right)^{2} \\
& \leq C_{2} \int_{0}^{T} \int_{\mathcal{D}} \hat{f}_{s}(x, \sigma)^{2} \underbrace{\leq}_{[66} C_{3} \int_{0}^{T} \int_{\mathcal{D}} 1+\left|u^{s}\right|^{2}+|u|^{2} .
\end{aligned}
$$

In view of (61), using (8) and uniform bounds on $f_{s}, \xi(s)$, we have

$$
\int_{\mathcal{D}} \kappa \xi(s)\left(u^{s}\right)^{2}(t) d x \leq t \int_{0}^{t}\left\|(\kappa \xi(s))^{\frac{1}{2}} f_{s}(\sigma) \xi(s)\right\|_{L^{2}(\mathcal{D})} d \sigma \leq C t \int_{0}^{t}\|f(\sigma)\|_{L^{2}(\mathcal{D})} d \sigma \quad \forall t \in[0, T],
$$

with $C$ independent of $s$ and $t$. Thus, using (68) and 69) we obtain

$$
\left\|q^{s}\right\|_{X}^{2} \leq C_{4} \int_{0}^{T}\|f(t)\|_{L^{2}(\mathcal{D})} d t \leq C_{5}
$$

where the constant $C_{5}$ does not depend on $s$, and consequently due to $q^{s}(t)=p^{s}(T-t)$ we also get $\left\|p^{s}\right\|_{X} \leq C$ for some constant $C$ independent of $s$.

Since $X$ is a Hilbert space, we can extract a weakly converging subsequence $p^{s} \rightarrow$ in $X$, using the uniform boundedness of $\left\|p^{s}\right\|_{X}$. Due to (59) and $\eta \in \mathcal{C}^{1}(\mathcal{D})$, we have the strong convergences $(\xi(s)-1) / s \rightarrow \operatorname{div} \theta$ in $\mathcal{C}(\mathcal{D})$ and $\left(\eta_{s} \xi(s)-\eta\right) / s \rightarrow \eta \operatorname{div} \theta+\nabla \eta \cdot \theta$ in $\mathcal{C}(\mathcal{D})$. Using $f \in L^{1}\left((0, T), H^{1}(\mathcal{D})\right)$, we also have $\left(f_{s} \xi(s)-f\right) / s \rightarrow$ $f \operatorname{div} \theta+\nabla f \cdot \theta$ in $L^{1}\left((0, T), L^{2}(\mathcal{D})\right)$, see [42, Section 2.14]. Using again (59) we obtain the strong convergence

$$
\lim _{s \searrow 0} \frac{A(s)-I}{s}=(\operatorname{div} \theta) R-D \theta R-R D \theta^{\top} \text { in } L^{\infty}\left(\mathcal{D}, \mathbb{R}^{n \times n}\right) .
$$

We now prove that

$$
\lim _{s \searrow 0} \frac{F\left(u, \Phi_{s}(x), t\right) \xi(s)-F(u, x, t)}{s} \rightarrow F(u, x, t) \operatorname{div}(\theta)+\nabla_{x} F(u, x, t) \cdot \theta \quad \text { in } L^{1}\left((0, T), L^{1}(\mathcal{D})\right) .
$$


Using a Taylor expansion we have

$$
w_{\rho}\left(\Phi_{s}(x)\right)=w_{\rho}(x)+s \nabla w_{\rho}(x) \cdot \theta+\left.\frac{s^{2}}{2} \frac{d^{2}}{d s^{2}}\left[w_{\rho}\left(\Phi_{s}(x)\right)\right]\right|_{s=\lambda}
$$

for some $\lambda \in[0, s]$. We compute using $(33)$ :

$$
\begin{aligned}
\frac{d^{2}}{d s^{2}}\left[w_{\rho}\left(\Phi_{s}(x)\right)\right] & =\nabla^{2} w_{\rho}\left(\Phi_{s}(x)\right)\left(\partial_{s} \Phi_{s}(x), \partial_{s} \Phi_{s}(x)\right)+\nabla w_{\rho}\left(\Phi_{s}(x)\right) \cdot \partial_{s}^{2} \Phi_{s}(x) \\
& =\nabla^{2} w_{\rho}\left(\Phi_{s}(x)\right)\left(\theta\left(\Phi_{s}(x)\right), \theta\left(\Phi_{s}(x)\right)\right)+\nabla w_{\rho}\left(\Phi_{s}(x)\right) \cdot D \theta\left(\Phi_{s}(x)\right) \theta\left(\Phi_{s}(x)\right) .
\end{aligned}
$$

Using $w_{\rho} \in \mathcal{C}^{2}(\overline{\mathcal{D}})$ and $\theta \in \mathcal{C}_{c}^{0,1}\left(\mathcal{D}, \mathbb{R}^{n}\right)$ we obtain

$$
\left\|\left.\frac{d^{2}}{d s^{2}}\left[w_{\rho}\left(\Phi_{s}(x)\right)\right]\right|_{s=\lambda}\right\|_{L^{\infty}(\mathcal{D})}<C,
$$

where $C$ is independent of $\lambda$. Using Assumption 3 we get

$$
\begin{aligned}
& \int_{0}^{T} \int_{\mathcal{D}}\left|\frac{F\left(u, \Phi_{s}(x), t\right) \xi(s)-F(u, x, t)}{s}-F(u, x, t) \operatorname{div}(\theta)-\nabla_{x} F(u, x, t) \cdot \theta\right| \\
& \leq \int_{0}^{T} \int_{\mathcal{D}}\left|\frac{F\left(u, \Phi_{s}(x), t\right)-F(u, x, t)}{s}-\nabla_{x} F(u, x, t) \cdot \theta\right|+\left|F(u, x, t) \frac{\xi(s)-1}{s}-F(u, x, t) \operatorname{div}(\theta)\right| \\
& +\int_{0}^{T} \int_{\mathcal{D}}\left|\left(F\left(u, \Phi_{s}(x), t\right)-F(u, x, t)\right) \frac{\xi(s)-1}{s}\right| \\
& \leq \frac{1}{2} \sum_{\rho=1}^{N_{\rho}} \underbrace{\left\|\frac{w_{\rho}\left(\Phi_{s}(x)\right)-w_{\rho}(x)}{s}-\nabla w_{\rho}(x) \cdot \theta\right\|_{L^{\infty}(\mathcal{D})}}_{\rightarrow 0 \text { due to }[71)-[72]} \underbrace{\int_{0}^{T} \int_{\mathcal{D}}\left(u-d_{\rho}\right)^{2}}_{<+\infty} \\
& +\frac{1}{2} \underbrace{\left\|\frac{\xi(s)-1}{s}-\operatorname{div}(\theta)\right\|_{L^{\infty}(\mathcal{D})}}_{\rightarrow 0 \text { due to } 55]} \sum_{\rho=1}^{N_{\rho}} \underbrace{\int_{0}^{T} \int_{\mathcal{D}}\left|w_{\rho}\right|\left(u-d_{\rho}\right)^{2}}_{<+\infty} \\
& +\frac{1}{2} \sum_{\rho=1}^{N_{\rho}} \underbrace{\left\|w_{\rho}\left(\Phi_{s}(x)\right)-w_{\rho}(x)\right\|_{L^{\infty}(\mathcal{D})}}_{\rightarrow 0} \underbrace{\left\|\frac{\xi(s)-1}{s}\right\|_{L^{\infty}(\mathcal{D})}}_{\rightarrow \operatorname{div}(\theta)} \underbrace{\int_{0}^{T} \int_{\mathcal{D}}\left(u-d_{\rho}\right)^{2}}_{<+\infty},
\end{aligned}
$$

where we have used the strong convergences $(\xi(s)-1) / s \rightarrow \operatorname{div} \theta$ in $\mathcal{C}(\mathcal{D}),\left(\Phi_{s}(x)-x\right) / s \rightarrow \theta$ in $\mathcal{C}\left(\mathcal{D}, \mathbb{R}^{n}\right)$, and also $d_{\rho} \in W^{1,1}((0, T), \mathbb{R}), w_{\rho} \in \mathcal{C}^{2}(\overline{\mathcal{D}})$ and $u \in \mathcal{C}\left([0, T], H_{\Gamma}^{1}(\mathcal{D})\right)$. This proves $[70)$.

Gathering the previous results and using (67) we have shown that

$$
\lim _{s \searrow 0} \frac{G\left(s, u, p^{s}\right)-G\left(0, u, p^{s}\right)}{s}=\partial_{s} G(0, u, p) .
$$

Thus, Assumption (H4) is satisfied and we can apply Theorem 3 This yields the shape derivative

$$
\begin{aligned}
& d J(\Omega)(\theta)=\partial_{s} G(0, u, p) \\
& =\int_{0}^{T} \int_{\mathcal{D}} F(u, x, t) \operatorname{div}(\theta)+\nabla_{x} F(u, x, t) \cdot \theta+\int_{0}^{T} \int_{\mathcal{D}}\left((\operatorname{div} \theta) R-D \theta R-R D \theta^{\top}\right) \nabla u \cdot \nabla p-\kappa u_{t} p_{t} \operatorname{div}(\theta) \\
& \quad+\int_{0}^{T} \int_{\mathcal{D}} \eta u_{t} p \operatorname{div}(\theta)+u_{t} p \nabla \eta \cdot \theta-f p \operatorname{div}(\theta)-p \nabla f \cdot \theta .
\end{aligned}
$$

Using the following tensor formulae

$$
D \theta R \nabla u \cdot \nabla p=D \theta:(\nabla p \otimes R \nabla u) \text { and } R D \theta^{\top} \nabla u \cdot \nabla p=D \theta:\left(\nabla u \otimes R^{\top} \nabla p\right),
$$

the fact that $R$ is symmetric, and $\operatorname{div} \theta=D \theta: I_{n}$, we obtain the distributed shape derivative in tensorial form (54). 
Using the assumption $f \in W^{1,1}\left((0, T), H^{1}(\mathcal{D})\right)$, Theorem 4 and Corollary 1 , we obtain the regularity

$$
u \in \mathcal{C}\left([0, T], H_{\Gamma}^{1}(\mathcal{D})\right) \cap \mathcal{C}^{2}\left([0, T], L^{2}(\mathcal{D})\right) \text { and } p \in \mathcal{C}\left([0, T], H_{\Gamma}^{1}(\mathcal{D})\right) \cap \mathcal{C}^{2}\left([0, T], L^{2}(\mathcal{D})\right) .
$$

Then, using $\eta \in \mathcal{C}^{1}(\mathcal{D})$ and the fact that $F$ satisfies the conditions of Assumption 3 , we obtain the regularity $\mathbf{S}_{1} \in L^{1}\left(\mathcal{D}, \mathbb{R}^{n \times n}\right)$ and $\mathbf{S}_{0} \in L^{1}\left(\mathcal{D}, \mathbb{R}^{n}\right)$ in view of the expressions 55$)$ and $(56)$ of $\mathbf{S}_{1}$ and $\mathbf{S}_{0}$.

\section{The Particular Case of FWI}

In Section 5 we have obtained a general expression for distributed shape derivatives of cost functionals depending on the solution of the acoustic wave equation with damping and discontinuous coefficients. The acoustic approximation in time-domain FWI fits into this general framework, with the following choice of parameters: $\mathcal{D}$ is a rectangle, the Neumann conditions correspond to the free surface of the Earth, and we choose $R=I_{n}$ in (1)-(5). This yields the following damped acoustic wave equation with discontinuous coefficient $\kappa$ :

$$
\begin{aligned}
\kappa u_{t t}-\Delta u+\eta u_{t} & =f \text { in } \mathcal{D} \times[0, T], \\
u(0) & =0 \text { in } \mathcal{D}, \\
u_{t}(0) & =0 \text { in } \mathcal{D}, \\
u & =0 \text { on } \Gamma \times[0, T], \\
\partial_{n} u & =0 \text { on } \Gamma_{n} \times[0, T],
\end{aligned}
$$

In this context, $u$ represents the acoustic pressure and $\kappa$ denotes the square slowness defined as $\kappa=1 / c^{2}$, where $c$ is the acoustic wave speed in the given physical media. Here $c=\sqrt{K / r}$, where $K$ is the bulk modulus and $r$ is the density; see 16 . Section 2.3] for a detailed discussion of this acoustic approximation. We assume that $f \in W^{1,1}\left((0, T), H^{1}(\mathcal{D})\right)$ and $\kappa \in L^{\infty}(\mathcal{D})$ is piecewise constant, i.e. $\kappa=\kappa_{\Omega}=\kappa_{0} \chi_{\Omega}+\kappa_{1} \chi_{\mathcal{D} \backslash \Omega}$ for some $\Omega \in \mathbb{P}(\mathcal{D})$, where $\kappa_{0}, \kappa_{1}>0$ are positive constants. In the context of FWI, the damping $\eta$ is used to prevent the reflection of waves on the artificial boundary $\Gamma$ in order to simulate an unbounded domain. In this case, $\eta$ is chosen equal to 0 inside the physical domain and positive inside a boundary layer, sometimes called sponge layer or damping mask, in the vicinity of the Dirichlet part $\Gamma$ of $\partial \mathcal{D}$; see Figure 2 for an illustration of the damping mask used in our numerical experiments.

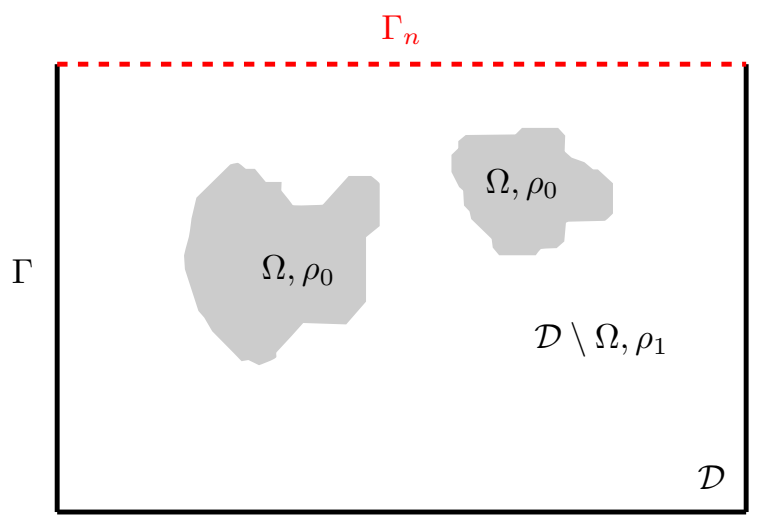

Figure 1. Partition $\mathcal{D}=\Omega \cup \Omega^{c}$

We assume that $N_{\rho}$ receivers are located at a set of points $x_{\rho} \in \Gamma_{n}$ for $\rho=1, \ldots, N_{\rho}$, i.e. the receivers are located on the surface. For $\rho=1, \ldots, N_{\rho}$, the seismograms $d_{\rho} \in W^{1, \infty}((0, T), \mathbb{R})$ denote the gathered data at these receivers. For the FWI application, the function $F: \mathbb{R} \times \mathbb{R}^{n} \times[0, T] \rightarrow \mathbb{R}$ in the shape optimization problem (31) is given by (30) with $w_{\rho}(x):=w\left(x-x_{\rho}\right)$, where $w$ is a mollifier of the Dirac measure at 0 . We assume that $w$ has compact support on a small open subset $\omega \subset \overline{\mathcal{D}}$.

Since the damping $\eta$ is concentrated on a boundary layer in the vicinity of $\Gamma$, and the source $f$ is concentrated near the surface $\Gamma_{n}$, we can make the following assumption. 
Assumption 4. The supports of $f, \eta$ and $\theta$ satisfy $\operatorname{supp}(\theta) \cap[\operatorname{supp}(\eta) \cup \operatorname{supp}(f)]=\emptyset$.

Under Assumptions 3 and 4 , and with the specific choice of parameters described at the beginning of this section to model the acoustic approximation used in FWI, the distributed shape derivative (54) is given by

$$
d J(\Omega)(\theta)=\int_{\mathcal{D}} \mathbf{S}_{1}: D \theta+\mathbf{S}_{0} \cdot \theta,
$$

with

$$
\begin{aligned}
& \mathbf{S}_{1}=\left[\int_{0}^{T}-\kappa u_{t} p_{t}+\nabla u \cdot \nabla p d t\right] I_{n}-\int_{0}^{T} \nabla u \otimes \nabla p+\nabla p \otimes \nabla u d t \\
& \mathbf{S}_{0}=0 .
\end{aligned}
$$

The adjoint satisfies (49)-(53), and with the parameters used for the acoustic approximation of FWI, 49) becomes in particular

$$
\kappa p_{t t}-\Delta p-\eta p_{t}=-\sum_{\rho=1}^{N_{\rho}} w_{\rho}\left(u-d_{\rho}\right) \text { in } \mathcal{D} \times[0, T] .
$$

\section{NUMERICAL IMPLEMENTATION}

For the numerical tests we take $\mathcal{D}=\{(x, z) \in[0,1] \times[0,0.65]\}$. Here, the Cartesian coordinates $(x, z)$ represent the position on the surface and the depth, respectively, i.e. $z=0$ corresponds to the surface. In the previous sections we have considered only one source $f$ to simplify the presentation of the results. In FWI, a set of point sources $\left\{f_{\sigma}\right\}_{\sigma=1}^{N_{\sigma}}$ is available, typically Ricker wavelets at various locations. In this case an acoustic pressure $u_{\sigma}$ and an adjoint $p_{\sigma}$ are computed for each source $f_{\sigma}$ and we simply sum the individual contributions of the cost functionals (30) over $\sigma=1, \ldots, N_{\sigma}$, i.e., we replace the objective functional of (31) by

$$
\frac{1}{2} \sum_{\sigma=1}^{N_{\sigma}} \sum_{\rho=1}^{N_{\rho}} \int_{0}^{T} \int_{\mathcal{D}} w_{\rho}(x)\left(u_{\sigma}(x, t)-d_{\rho, \sigma}(t)\right)^{2} d x d t,
$$

where $d_{\rho, \sigma}$ denotes the seismogram corresponding to $f_{\sigma}$ and the receiver at $x_{\rho}$.

The global shape derivative is then also the sum of the expressions 78 over $\sigma=1, \ldots, N_{\sigma}$. Also, from the perspective of the FWI application, it is natural to assume that the support of the mollifier $w_{\rho}$ is smaller than the grid size so that, from a numerical viewpoint, $w_{\rho}$ is indistinguishable from a Dirac measure at $x_{\rho}$. In this case, the numerical discretization of 82 approximates

$$
\frac{1}{2} \sum_{\sigma=1}^{N_{\sigma}} \sum_{\rho=1}^{N_{\rho}} \int_{0}^{T}\left(u_{\sigma}\left(x_{\rho}, t\right)-d_{\rho, \sigma}(t)\right)^{2} d t,
$$

which is typically used in FWI as the objective functional.

In order to obtain a smooth descent direction $\theta$, i.e. a vector field satisfying $d J(\Omega)(\theta)<0$, we solve the elliptic equation: find $\theta \in H_{0}^{1}(\mathcal{D})^{2}$ such that

$$
\int_{\mathcal{D}} \alpha_{1} g D \theta: D \xi+\alpha_{2} g \theta \cdot \xi d x=-d J(\Omega)(\xi) \quad \text { for all } \xi \in H_{0}^{1}\left(\mathcal{D}, \mathbb{R}^{2}\right),
$$

which means

$$
\int_{\mathcal{D}} \alpha_{1} g D \theta: D \xi+\alpha_{2} g \theta \cdot \xi d x=-\int_{\mathcal{D}} \mathbf{S}_{1}: D \xi+\mathbf{S}_{0} \cdot \xi \quad \text { for all } \xi \in H_{0}^{1}\left(\mathcal{D}, \mathbb{R}^{2}\right),
$$

where $\alpha_{1}=0.01, \alpha_{2}=0.97$. The function $g: \mathbb{R}^{2} \mapsto \mathbb{R}$ is designed to be almost constant inside $\mathcal{D}$ and take large values close to the boundary, in order to force $\theta$ to take small values close to the boundary, and in particular in the damping layer where $\eta$ is positive.

The evolution of the domain is modeled via a level set method, introduced in 38]. The key idea of this numerical method is to implicitly represent the boundary of the moving domain $\overline{\Omega_{s}} \subset \mathcal{D} \in \mathbb{R}^{N}$ as the zero 

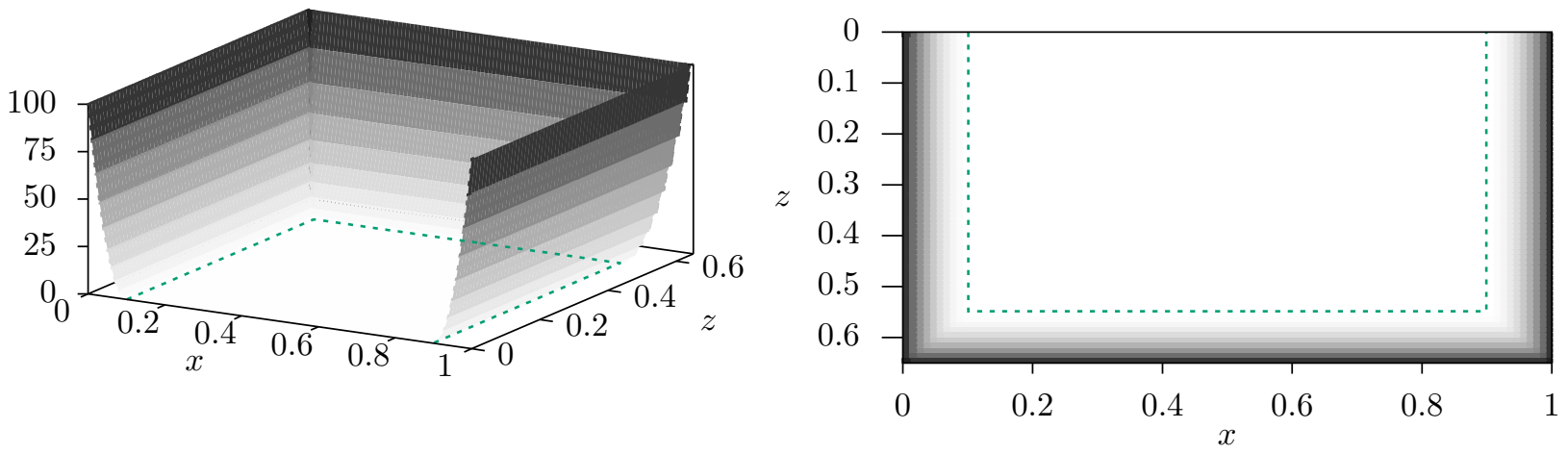

Figure 2. 3D view (left) and 2D view (right) of the damping mask $\eta$. In the physical domain, whose limits are represented by the dashed lines, we have $\eta \equiv 0$, while $\eta$ is large close to the artificial boundary $\Gamma$ in order to mitigate wave reflections.

level set of a Lipschitz continuous function $\phi: \mathcal{D} \times \mathbb{R}^{+} \rightarrow \mathbb{R}$. A family of moving domains $\Omega_{s} \subset \mathcal{D}$ is defined as

$$
\Omega_{s}:=\{(x, z) \in \mathcal{D} \mid \phi(x, z, s)<0\}, \quad \text { so that } \quad \partial \Omega_{s}=\{(x, z) \in \mathcal{D} \mid \phi(x, z, s)=0\},
$$

where we assume $|\nabla \phi(\cdot, s)| \neq 0$ on $\partial \Omega_{s}$. It can be shown that the evolution of $\phi$ depends on the descent direction $\theta$ through the following transport equation:

$$
\partial_{s} \phi(x, z, s)+\theta(x, z) \cdot \nabla \phi(x, z, s)=0 \text { in } \mathcal{D} \times \mathbb{R}^{+} .
$$

The algorithm consists in first calculating $\theta$ using (83), and then to solve (84) to update the domain $\Omega_{s}$. We use a Lax-Friedrichs flux for the discretization of (84), and refer to 28 for implementation details.

To simulate noisy seismic data, each synthetic seismogram $d_{\rho, \sigma}(t)$ is first generated using the ground truth, and then corrupted by adding a normal Gaussian noise with mean zero and standard deviation $\delta \cdot\left\|d_{\rho, \sigma}\right\|_{\infty}$, where $\delta$ is a parameter. Let $d_{\rho, \sigma}$ and $\tilde{d}_{\rho, \sigma}$ denote respectively the noiseless and noisy seismograms corresponding to the source $f_{\sigma}$ and recorded at the receiver $\rho$. The noise level is then computed as

$$
\text { noise level }=\left[\frac{\int_{0}^{T} \sum_{\sigma=1}^{N_{\sigma}} \sum_{\rho=1}^{N_{\rho}}\left|d_{\rho, \sigma}(t)-\tilde{d}_{\rho, \sigma}(t)\right|^{2}}{\int_{0}^{T} \sum_{\sigma=1}^{N_{\sigma}} \sum_{\rho=1}^{N_{\rho}}\left|d_{\rho, \sigma}(t)\right|^{2}}\right]^{1 / 2} .
$$

We present three numerical experiments with $c_{0}=4.12 \mathrm{~km} / \mathrm{s}$ and $c_{1}=1.95 \mathrm{~km} / \mathrm{s}$, with $\kappa_{0}=1 / c_{0}^{2}$ and $\kappa_{1}=1 / c_{1}^{2}$. These specific values of $c_{0}, c_{1}$ are based on common geophysics standards, and correspond to real data of wave speed propagation inside salt and sediments; respectively. The domain $\mathcal{D}$ is a rectangle of length $1 \mathrm{~km}$ in $x$-axis and depth $0.65 \mathrm{~km}$ in $z$-axis, which is meshed using a regular grid with $n_{x} \times n_{z}$ grid points.

The wave equations (73)-(77) are solved using a second-order explicit finite-difference scheme. The time step $\Delta t$ is constrained by the CFL condition

$$
\Delta t \leq \frac{a}{c_{\max }\left(\Delta x^{-1}+\Delta z^{-1}\right)}
$$

with the grid steps $\Delta x=1 / n_{x}$ and $\Delta z=0.65 / n_{z}, c_{\max }=\max \{c(x) \mid x \in \mathcal{D}\}$ is the maximum of the wave speed inside the domain $\mathcal{D}$, and $a$ is the Courant number equal to 0.4 in our numerical experiments.

In all three numerical experiments, we use $N_{\sigma}=10$ shots modeled by Ricker wavelets with dominant frequency of $5 \mathrm{~Hz}$ to simulate the data acquisition, and $N_{\rho}=80$ receivers placed on the surface $\Gamma_{n}$ with a spacing of $0.01 \mathrm{~km}$ between the receivers. The shots and receivers are placed at grid points for simplicity. We use a regular grid with $n_{x} \times n_{z}=200 \times 130$ grid points, and synthetic seismograms of $T=2$ seconds are recorded at the receivers using the ground truth. 

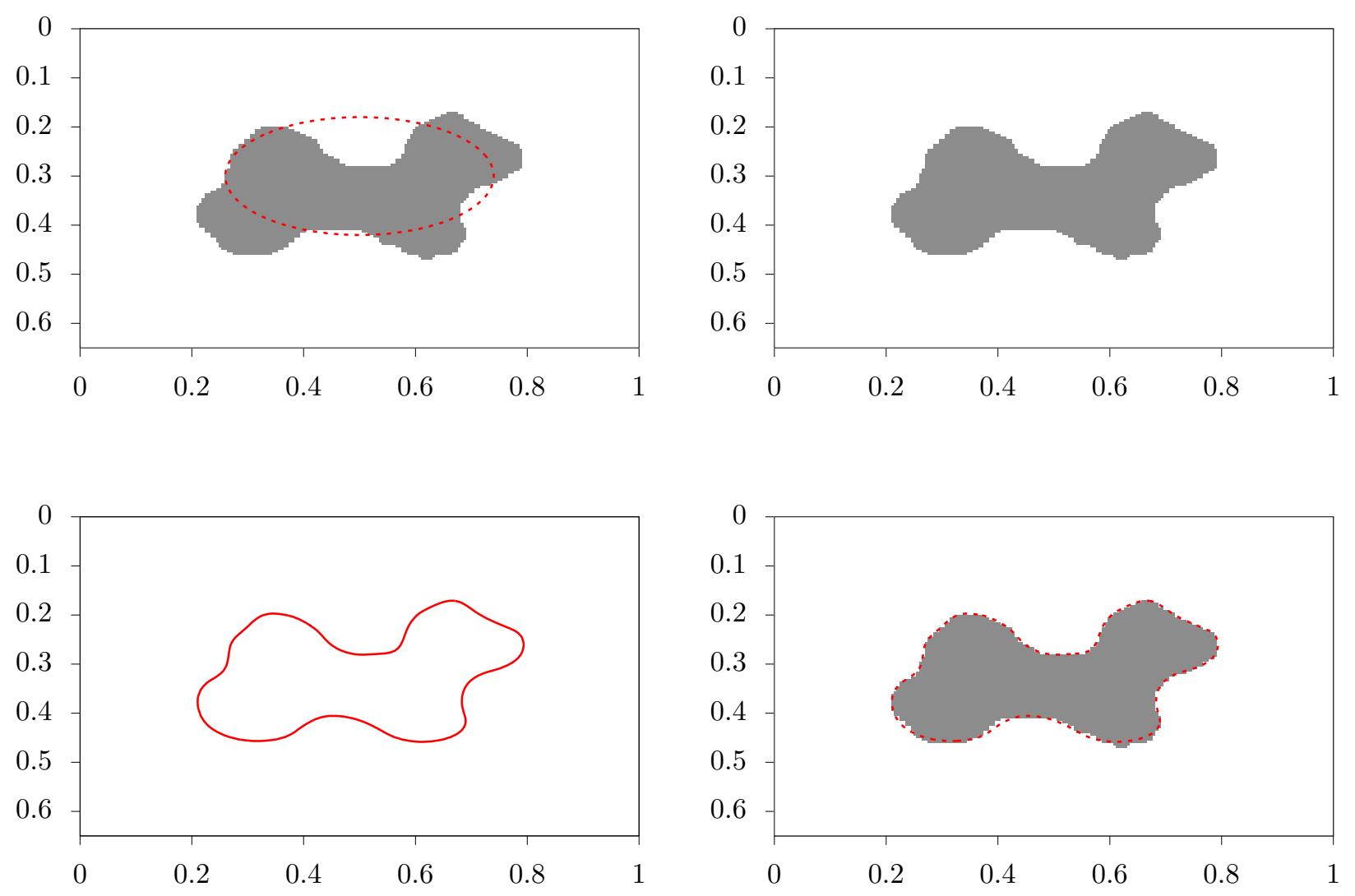

FiguRE 3. Reconstruction of one inclusion, using 10 shots with Ricker wavelet with dominant frequency of $5 \mathrm{~Hz}, 80$ receivers placed on the surface $\Gamma_{n}$, a $200 \times 130$ grid, and a noise level of $2.05 \%$. Superposition of ground truth (gray shape) and dashed contour of the initialization (top left), ground truth (top right), contour of the reconstructed shape $\Omega$ (bottom left) and superposition of the reconstruction and of the ground truth (bottom right).

In the first experiment, the ground truth consists of one block representing the salt body and we initialize $\Omega$ using a large ellipse; see Figure 3 . In the second experiment, the ground truth consists of two disconnected blocks representing two salt bodies and we initialize $\Omega$ using two small disks; see Figure 4 . In the third experiment, the ground truth consists of three disconnected blocks representing three salt bodies and we initialize $\Omega$ using three small disks; see Figure 5. We observe that in all three experiments the interface reconstruction is very accurate in the upper region of the salt body. In the first experiment (Figure 3 ) the reconstruction is also very accurate in the lower part of the salt body, although small defects can be observed. In the second and third experiments (Figures 4 and 5 , the reconstruction is still reasonably accurate in the lower region but the defects are visibly larger than in the first experiment. These inaccuracies were expected due to the lack of illumination of the lower parts of the salt bodies and are standard in FWI.

These numerical results show that the method is capable of accurate reconstructions in the framework of piecewise constant velocities. A line for future research consists in filling the gap towards more realistic applications. In particular, this includes applications to large-scale 2D and 3D problems, and extending the method to handle more complex structures such as piecewise smooth reconstructions.

Acknowledgements. Yuri Flores Albuquerque and Antoine Laurain gratefully acknowledge support of the RCGI - Research Centre for Gas Innovation, hosted by the University of São Paulo (USP) and sponsored 

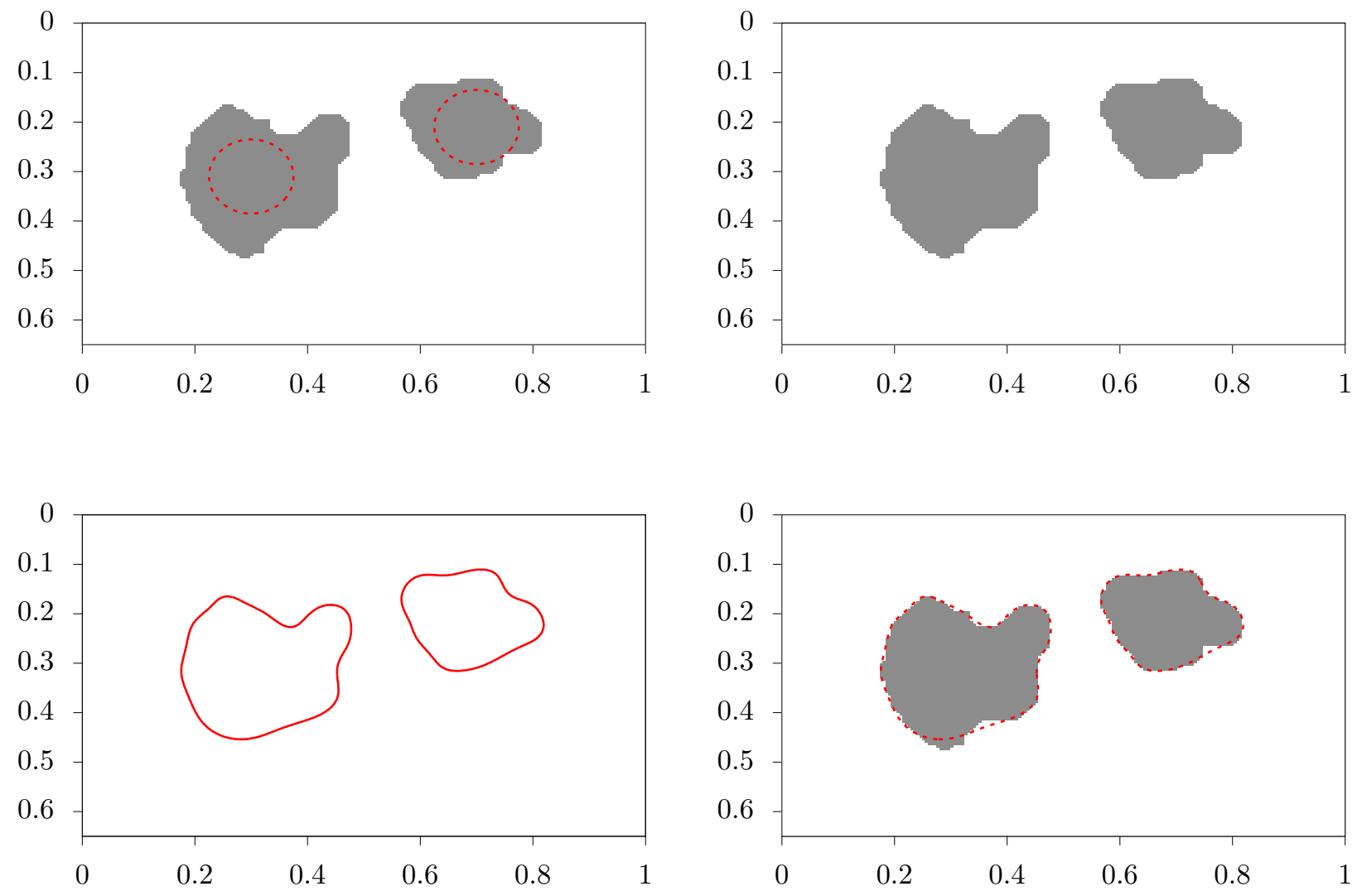

FiguRE 4. Reconstruction of two inclusions, using 10 shots with Ricker wavelet with dominant frequency of $5 \mathrm{~Hz}, 80$ receivers placed on the surface $\Gamma_{n}$, a $200 \times 130$ grid, with a noise level of $2.0 \%$. Superposition of ground truth (gray shape) and dashed contour of the initialization (top left), ground truth (top right), contour of the reconstructed shape $\Omega$ (bottom left) and superposition of the reconstruction and of the ground truth (bottom right).

by FAPESP - São Paulo Research Foundation (2014/50279-4) and Shell Brasil. This research was carried out in association with the ongoing R\&D project registered as ANP 20714-2 - Desenvolvimento de técnicas numéricas e software para problemas de inversão com aplicações em processamento sísmico (USP / Shell Brasil / ANP), sponsored by Shell Brasil under the ANP R\&D levy as "Compromisso de Investimentos com Pesquisa e Desenvolvimento". Antoine Laurain gratefully acknowledges the support of the Brazilian National Council for Scientific and Technological Development (Conselho Nacional de Desenvolvimento Científico e Tecnológico - CNPq) through the process: 408175/2018-4 "Otimização de forma não suave e controle de problemas de fronteira livre", and through the program "Bolsa de Produtividade em Pesquisa - PQ 2015", process: 304258/2018-0. The work of Irwin Yousept was supported by the German Research Foundation Priority Programm DFG SPP 1962 "Non-smooth and Complementarity-based Distributed Parameter Systems: Simulation and Hierarchical Optimization", Project YO 159/2-2.

\section{REFERENCES}

[1] H. S. Aghamiry, A. Gholami, and S. Operto. Implementing bound constraints and total-variation regularization in extended full waveform inversion with the alternating direction method of multiplier: application to large contrast media. Geophysical Journal International, Apr. 2019. 

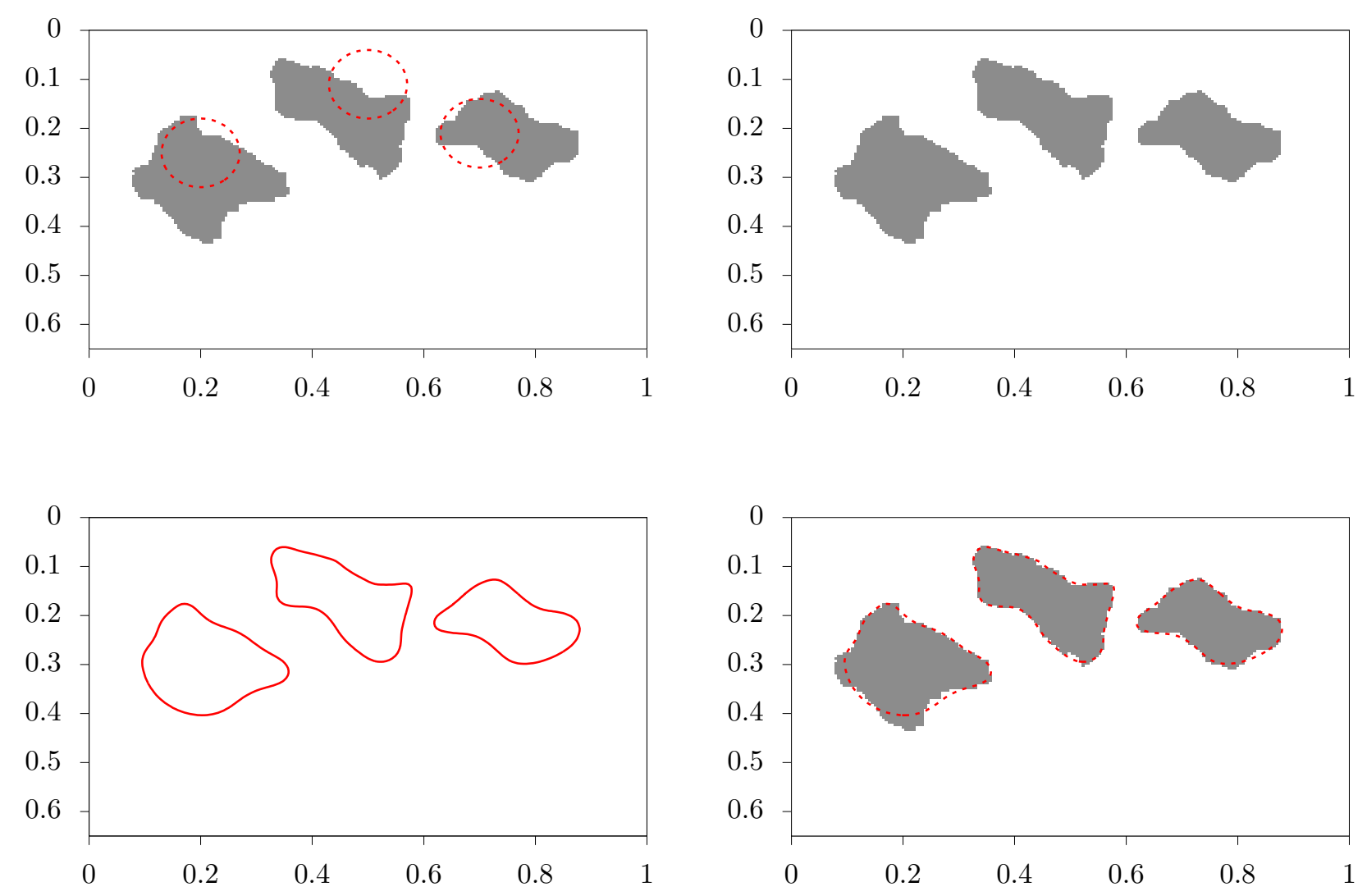

FiguRE 5. Reconstruction of three inclusions, using 10 shots with Ricker wavelet with dominant frequency of $5 \mathrm{~Hz}, 80$ receivers placed on the surface $\Gamma_{n}$, a $200 \times 130$ grid, and a noise level of $1.88 \%$. Superposition of ground truth (gray shape) and dashed contour of the initialization (top left), ground truth (top right), contour of the reconstructed shape $\Omega$ (bottom left), superposition of the reconstruction and of the ground truth (bottom right).

[2] Y. Albuquerque and A. Laurain. Reconstruction of sharp interfaces in time-domain full waveform inversion. In Conference Proceedings, 82nd EAGE Annual Conference \& Exhibition Workshop Programme, pages 1-5. European Association of Geoscientists \& Engineers, 2020.

[3] H. Ammari. An inverse initial boundary value problem for the wave equation in the presence of imperfections of small volume. SIAM Journal on Control and Optimization, 41(4):1194-1211, Jan. 2002.

[4] J. M. Ball. Strongly continuous semigroups, weak solutions, and the variation of constants formula. Proc. Amer. Math. Soc., 63(2):370-373, 1977.

[5] G. Bao and W. W. Symes. On the sensitivity of solutions of hyperbolic equations to the coefficients. Communications in Partial Differential Equations, 21(3-4):395-422, 1996.

[6] E. Beretta, M. V. de Hoop, E. Francini, S. Vessella, and J. Zhai. Uniqueness and lipschitz stability of an inverse boundary value problem for time-harmonic elastic waves. Inverse Problems, 33(3):035013, feb 2017.

[7] C. Boehm. Efficient Inversion Methods for Constrained Parameter Identification in Full-Waveform Seismic Tomography. $\mathrm{PhD}$ thesis, Technische Universität München, 2015.

[8] C. Boehm and M. Ulbrich. A semismooth Newton-CG method for constrained parameter identification in seismic tomography. SIAM J. Sci. Comput., 37(5):S334-S364, 2015.

[9] J. Cagnol and J.-P. Zolésio. Shape derivative in the wave equation with Dirichlet boundary conditions. J. Differential Equations, 158(2):175-210, 1999.

[10] M. Cea. Optimal design for 2d wave equations. Optimization Methods and Software, 32(1):86-108, 2017.

[11] D.-H. Chen and I. Yousept. Variational source condition for ill-posed backward nonlinear Maxwell's equations. Inverse Problems, 35(2):25001, 25, 2019. 
[12] C. Clason, K. Kunisch, and P. Trautmann. Optimal control of the principal coefficient in a scalar wave equation, 2020, arXiv:1912.08672v2.

[13] D. Datta, M. K. Sen, F. Liu, and S. Morton. Full-waveform inversion of salt models using shape optimization and simulated annealing. Geophysics, 84(5):R793-R804, 092019.

[14] M. Delfour, G. Payre, and J.-P. Zolésio. An optimal triangulation for second-order elliptic problems. Comput. Methods Appl. Mech. Engrg., 50(3):231-261, 1985.

[15] E. Esser, L. Guasch, T. van Leeuwen, A. Y. Aravkin, and F. J. Herrmann. Total variation regularization strategies in full-waveform inversion. SIAM Journal on Imaging Sciences, 11(1):376-406, Jan. 2018.

[16] A. Fichtner. Full Seismic Waveform Modelling and Inversion. Springer Berlin Heidelberg, 2011.

[17] Z. Guo and M. V. de Hoop. Shape optimization in full waveform inversion with sparse blocky model representations. Proceedings of the Project Review, 1:189-208, 2012.

[18] Z. Guo and M. V. de Hoop. Shape optimization and level set method in full waveform inversion with 3d body reconstruction. In SEG Technical Program Expanded Abstracts 2013, pages 1079-1083. Society of Exploration Geophysicists, 2013.

[19] P. Hébrard and A. Henrot. A spillover phenomenon in the optimal location of actuators. SIAM J. Control Optim., 44(1):349366, July 2005.

[20] M. Hintermüller, A. Laurain, and I. Yousept. Shape sensitivities for an inverse problem in magnetic induction tomography based on the eddy current model. Inverse Problems, 31(6):065006, 25, 2015.

[21] R. Hiptmair, A. Paganini, and S. Sargheini. Comparison of approximate shape gradients. BIT Numerical Mathematics, 55(2):459-485, Aug. 2014.

[22] A. Kadu, T. van Leeuwen, and W. A. Mulder. Salt reconstruction in full-waveform inversion with a parametric level-set method. IEEE Transactions on Computational Imaging, 3(2):305-315, June 2017.

[23] A. Kirsch. The domain derivative and two applications in inverse scattering theory. Inverse Problems, 9(1):81-96, 1993.

[24] A. Kirsch and A. Rieder. On the linearization of operators related to the full waveform inversion in seismology. Math. Methods Appl. Sci., 37(18):2995-3007, 2014.

[25] A. Kirsch and A. Rieder. Inverse problems for abstract evolution equations with applications in electrodynamics and elasticity. Inverse Problems, 32(8):085001, jun 2016.

[26] K. F. Lam and I. Yousept. Consistency of a phase field regularisation for an inverse problem governed by a quasilinear Maxwell system. Inverse Problems, 36(4):045011, 33, 2020.

[27] D. Lanzsnaster, P. B. de Castro, H. E. Junior, P. T. R. Mendoca, E. C. N. Silva, and E. A. Fancello. A level-set approach based on reaction-diffusion equation applied to inversion problems in acoustic wave propagation. Inverse Problems, Oct. 2020.

[28] A. Laurain. A level set-based structural optimization code using FEniCS. Structural and Multidisciplinary Optimization, 58(3):1311-1334, May 2018.

[29] A. Laurain. Distributed and boundary expressions of first and second order shape derivatives in nonsmooth domains. Journal de Mathématiques Pures et Appliquées, 134:328 - 368, 2020.

[30] A. Laurain and K. Sturm. Distributed shape derivative via averaged adjoint method and applications. ESAIM Math. Model. Numer. Anal., 50(4):1241-1267, 2016.

[31] A. Lechleiter and J. W. Schlasche. Identifying Lamé parameters from time-dependent elastic wave measurements. Inverse Problems in Science and Engineering, 25(1):2-26, 2017.

[32] W. Lewis, B. Starr, and D. Vigh. A level set approach to salt geometry inversion in full-waveform inversion. In SEG Technical Program Expanded Abstracts 2012, pages 1-5. Society of Exploration Geophysicists, 2012.

[33] W. Lewis and D. Vigh. 3D salt geometry inversion in full-waveform inversion using a level-set method, pages 1221-1226. Society of Exploration Geophysicists, 2016.

[34] A. Münch. Optimal design of the support of the control for the 2-D wave equation: a numerical method. International Journal of Numerical Analysis and Modeling, 5(2):331-351, 2008.

[35] A. Münch. Optimal internal dissipation of a damped wave equation using a topological approach. International Journal of Applied Mathematics and Computer Science, 19(1):15 - 38, 2009.

[36] A. Münch, P. Pedregal, and F. Periago. Optimal design of the damping set for the stabilization of the wave equation. Journal of Differential Equations, 231(1):331-358, Dec. 2006.

[37] A. Nowakowski. Shape optimization of control problems described by wave equations. Control Cybernet., 37(4):1045-1055, 2008.

[38] S. Osher and J. A. Sethian. Fronts propagating with curvature-dependent speed: algorithms based on Hamilton-Jacobi formulations. J. Comput. Phys., 79(1):12-49, 1988.

[39] A. Pazy. Semigroups of Linear Operators and Applications to Partial Differential Equations. Springer-Verlag, New York, 1983.

[40] Y. Privat, E. Trélat, and E. Zuazua. Optimal location of controllers for the one-dimensional wave equation. Annales de l'Institut Henri Poincare (C) Non Linear Analysis, 30(6):1097 - 1126, 2013.

[41] J. Shi, R. Ye, and M. de Hoop. Full-waveform inversion with 3D-shape optimization on unstructured meshes, pages 15281532. Society of Exploration Geophysicists, 2017.

[42] J. Sokołowski and J.-P. Zolésio. Introduction to shape optimization, volume 16 of Springer Series in Computational Mathematics. Springer-Verlag, Berlin, 1992. Shape sensitivity analysis.

[43] C. Stolk. On the modeling and inversion of seismic data. PhD thesis, University of Utrecht, The Netherlands, 2000. Available from https://dspace.library.uu.nl/handle/1874/855. 
[44] K. Sturm. Minimax Lagrangian approach to the differentiability of nonlinear PDE constrained shape functions without saddle point assumption. SICON, 53(4):2017-2039, 2015.

[45] J. Virieux and S. Operto. An overview of full-waveform inversion in exploration geophysics. GEOPHYSICS, 74(6):WCC1WCC26, Nov. 2009.

[46] I. Yousept. Finite element analysis of an optimal control problem in the coefficients of time-harmonic eddy current equations. J. Optim. Theory Appl., 154(3):879-903, 2012.

[47] I. Yousept. Hyperbolic Maxwell variational inequalities of the second kind. ESAIM Control Optim. Calc. Var., 26:Paper No. 34, 2020.

[48] I. Yousept. Well-posedness theory for electromagnetic obstacle problems. J. Differential Equations, 269(10):8855-8881, 2020.

[49] W. P. Ziemer. Weakly Differentiable Functions. Springer New York, 1989.

(Yuri Flores Albuquerque) Instituto de Matemática e Estatística, Universidade de São Paulo, Rua do Matão 1010 , 05508-090, SÃo Paulo, Brazil

E-mail address: yuri.falbu@gmail.com

(Antoine Laurain) Instituto de Matemática e Estatística, Universidade de São Paulo, Rua do Matão 1010, 05508090, SÃo PAUlo, BRAZIL

E-mail address: laurain@ime.usp.br

(Irwin Yousept) University of Duisburg-Essen, Fakultät für Mathematik, Thea-Leymann-Str. 9, D-45127 Essen, GeRmany

E-mail address: irwin.yousept@uni-due.de 